\title{
Numerical Modeling of Diffusive Heat Transport Across Magnetic Islands and Local Stochastic Field
}

\author{
Q. Yu \\ Max-Planck-Institut für Plasmaphysik, EURATOM Association, D-85748 Garching, Germany
}

The heat diffusion across magnetic islands is studied numerically and compared with analytical results. For a single island, the enhanced radial heat diffusivity, $\chi_{\mathrm{r}}$, due to the parallel transport along the field lines is increased over a region of about the island width $\mathrm{w}$. The maximum enhanced heat conductivity at the rational surface is proportional to $\mathrm{w}^{2}\left(\chi_{\|} \chi_{\perp}\right)^{1 / 2}$ for sufficiently high values of $\chi_{\|} / \chi_{\perp}$, where $\chi_{\|} / \chi_{\perp}$ is the ratio between the parallel and the perpendicular heat diffusivity. For low ratios of $\chi_{\| 1} \chi_{\perp}$, however, the maximum value of $\chi_{\mathrm{r}}$ is proportional to $\mathrm{w}^{4} \chi_{\|}$. In a locally stochastic magnetic field, $\chi_{\mathrm{r}}$ is again proportional to $\mathrm{w}^{4} \chi_{\|}$for low $\chi_{\|} / \chi_{\perp}$, which is in agreement with the analytical results. With increasing $\chi_{\|} / \chi_{\perp}, \chi_{\mathrm{r}}$ is dominated first by the additive effect of individual islands and then by the field ergodicity. 


\section{Introduction}

Magnetic islands generally exist in laboratory and space plasmas, caused either by resonant helical magnetic field perturbations (finite error fields) in fusion devices or by the tearing mode type instabilities, driven by an unfavorable plasma current density gradient [1-3], the perturbed bootstrap current [4-9], or the electron temperature gradient $[10,11]$. The existence of magnetic islands inside the plasma usually has a very significant effect on the plasma behavior. In tokamak plasmas magnetic islands caused by the classical or the neoclassical tearing mode (NTM) have been found to lead to a degradation of plasma confinement or even to disruptions [2-8]. For high $\beta$ plasmas the onset of a magnetic field perturbation with its corresponding rational surface being very close to another saturated island is often observed, and a strong interaction between them and the subsequent change in the plasma energy confinement are found [6,12]. Extensive studies have also been devoted to the transport across the stochastic field boundary in stellarators and in tokamaks as produced by an externally applied helical field $[13,14]$. The heat transport across magnetic islands and the stochastic magnetic field is thus of general interest in plasma physics.

The effect of a single magnetic island on the plasma energy confinement has been analyzed by assuming the ratio between the parallel and the perpendicular heat conductivity, $\chi_{\|} / \chi_{\perp}$, to be infinite. The resulting degradation of plasma energy confinement due to the fast parallel transport along the field lines is found to be determined by the island width, the minor radius of the rational surface and the local equilibrium plasma pressure gradient [15]. The electron temperature perturbations due to a single island have also been studied in the limits of a sufficiently low or high value of $\chi_{\|} / \chi_{\perp}$ for determining the transport threshold for the onset of the NTM [16]. When the plasma region is occupied by well-overlapping islands, the magnetic field becomes stochastic there. For such a situation the enhanced radial 
transport across the stochastic field has been extensively investigated and is considered to be one of the possible mechanisms for the anomalous heat transport of tokamak plasmas [17-26].

It is well understood that both the parallel and the perpendicular transport are important in determining the heat diffusion across a single island or a stochastic field, no matter if the perpendicular transport is caused by plasma collisions or turbulence [16-20]. Despite the existing theories on the heat transport across stochastic fields, for a finite value of $\chi_{\|} / \chi_{\perp}$ there is no straightforward analytical solution for the temperature perturbations even due to a single island [16]. Since the plasma temperature changes by several orders of magnitude from the center to the edge in a tokamak, the parameter $\chi_{\|} / \chi_{\perp}$ changes even more. It is of great interest to study how the parameter $\chi_{\|} / \chi_{\perp}$ affects the heat transport across islands and the related plasma energy confinement degradation. In some experimental cases one finds a local stochastic field region due to magnetic perturbations of different helicities with their corresponding rational surfaces being close together $[6,12]$. To the author's knowledge no transport theory has been established for such a local stochastic field yet. The understanding of the heat transport in such a case is however important for understanding both the corresponding change in the plasma energy confinement as well as its effect on plasma instabilities such as the NTMs $[6,12]$. The drift-tearing mode instability driven by the electron temperature gradient is found to be particularly sensitive to the perpendicular heat transport [11].

The numerical calculation of the heat transport across magnetic islands is usually quite challenging for a high ratio of $\chi_{\|} / \chi_{\perp}$, which could be in the order of $10^{10}$ or even larger in the central region of large tokamak plasmas [16]. This large $\chi_{\|} / \chi_{\perp}$ usually introduces an artificial perpendicular heat flux which could be even larger than the real one. Recently a new numerical method was developed, which has been shown to suppress this artificial perpendicular heat flux [27]. In addition to the numerical problem, the parallel heat flux is also much more 
complicated for a sufficiently high $\chi_{\|} / \chi_{\perp}$. It is of the classical form only in a narrow region around the rational surface or the island's x-point [28]. Away from this region the heat flux is carried by free-streaming electrons and is non-local [29]. For such a case the "flux limit" form for $\chi_{\|}$was used before [e.g., 16, 30], but this is only a crude approximation [29].

In the present paper the complicated physics of the parallel heat flux mentioned above will not be addressed. Our numerical results of the diffusive heat transport across magnetic islands are obtained by using a constant $\chi_{\|}$along the minor radius. This assumption is the same as that of the existing theories, allowing an easy comparison with them [16-20]. For a single island, the enhanced radial heat diffusivity $\chi_{\mathrm{r}}$ due to the parallel transport along the field lines is found to be increased over a radial width of about the island width w. The maximum value at the rational surface is proportional to $\mathrm{w}^{2}\left(\chi_{\|} \chi_{\perp}\right)^{1 / 2}$ for a sufficiently high value of $\chi_{\|} / \chi_{\perp}$. For a low ratio of $\chi_{\|} / \chi_{\perp}, \chi_{\mathrm{r}}$ is proportional to $\mathrm{w}^{4} \chi_{\|}$. When two neighboring islands overlap, the local magnetic field becomes stochastic. In this case, for low ratios of $\chi_{\|} / \chi_{\perp}$, the radial heat transport is found to be dominated by the additive effect of individual islands. This is in agreement with the analytical result as shown in the Appendix. Only for sufficiently high ratios of $\chi_{\|} / \chi_{\perp}$, the radial heat transport is dominated by the ergodicity of the magnetic field.

In the following Section II our model is described. The numerical results and the comparison with analytical results are presented in Section III, and the discussion and summary are given in Section V.

\section{Model}

In our model the large aspect-ratio tokamak approximation is utilized. The magnetic field

$\mathbf{B}$ is expressed in the form $\mathbf{B}=\mathrm{B}_{0 \mathrm{t}} \mathbf{e}_{\mathrm{t}}+\mathrm{B}_{0 \mathrm{p}}(\mathrm{r}) \mathbf{e}_{\mathrm{p}}+\mathbf{B}_{1}$, where $\mathrm{B}_{0 \mathrm{t}}$ and $\mathrm{B}_{0 \mathrm{p}}$ are the equilibrium 
toroidal and poloidal magnetic field, respectively, and $\mathbf{B}_{1}=\nabla \psi \times \mathbf{e}_{\mathrm{t}}$ is the perturbed helical field. $\mathrm{B}_{0 \mathrm{t}}$ is assumed to be a constant. $\psi$ is expressed in terms of a Fourier decomposition,

$$
\psi=\sum_{i} \psi_{i}(r) \cos \left(m_{i} \theta+n_{i} \phi\right)
$$

where $\mathrm{m}_{\mathrm{i}}$ and $\mathrm{n}_{\mathrm{i}}$ are the poloidal and toroidal mode numbers of the $i$ th component of $\mathbf{B}_{1}$, and $\theta$ and $\phi$ are the poloidal and toroidal angle, respectively. The toroidal magnetic field perturbation is usually much smaller than the poloidal one and is therefore neglected.

The equilibrium safety factor $\mathrm{q}(\mathrm{r})=\mathrm{rB}_{0 \mathrm{r}} /\left(\mathrm{RB}_{0 \mathrm{p}}\right)$ is chosen to be the form

$$
\mathrm{q}(\mathrm{r})=\mathrm{q}_{0} e^{r / L_{q}}
$$

to have a constant magnetic shear along the minor radius, where $\mathrm{q}_{0}=0.2$ and $\mathrm{L}_{\mathrm{q}}=0.3 \mathrm{a}$ are chosen to ensure $\mathrm{q}=3 / 2$ and $4 / 3$ surfaces to be inside the plasma $(0 \leq \mathrm{r} \leq \mathrm{a}$, a: plasma minor radius), except when mentioned elsewhere.

. $\psi_{\mathrm{i}}(\mathrm{r})$ in Eq. (1) is taken as

$$
\psi_{i}(r)=\psi_{i, 0}(r / a)^{2}(1-r / a)^{2}
$$

to have a smooth change along the minor radius, being typical for the classical or neoclassical tearing modes $[1,9]$.

The following electron energy transport equation

$$
\frac{3}{2} \mathrm{n}_{\mathrm{e}} \frac{\partial \mathrm{T}_{\mathrm{e}}}{\partial \mathrm{t}}=\mathrm{n}_{\mathrm{e}} \nabla \cdot\left(\chi_{\|} \nabla_{\|} \mathrm{T}_{\mathrm{e}}\right)+\mathrm{n}_{\mathrm{e}} \nabla \cdot\left(\chi_{\perp} \nabla_{\perp} \mathrm{T}_{\mathrm{e}}\right)+\mathrm{P}(\mathrm{r})
$$

is solved, where $T_{e}$ is the electron temperature, $n_{e}$ is the electron density and $P$ is the heat source. Here $n_{e}, \chi_{\|}$and $\chi_{\perp}$ are assumed to be constant along the minor radius for simplicity, and the convective transport is neglected which is valid for slowly changing islands such as those due to an error field or NTMs $[9,16]$.

The heat source $\mathrm{P}(\mathrm{r})$ in Eq. (4) is taken to be of the form 


$$
\mathrm{P}(\mathrm{r})=\mathrm{P}_{0}\left[1-\left(\frac{r}{a}\right)^{c_{1}}\right]^{c_{2}}
$$

where $c_{1}=2$ and $c_{2}=16$ are taken except when mentioned elsewhere. $P_{0}$ is chosen to ensure $\mathrm{T}_{\mathrm{e} 0}(\mathrm{r}=0)=1$, where $\mathrm{T}_{\mathrm{e} 0}$ is the equilibrium electron temperature obtained from Eq. (4) by taking $\mathbf{B}_{1}=0$ (without magnetic field perturbations).

The boundary conditions used here are $\mathrm{T}_{\mathrm{e}}(\mathrm{r}=\mathrm{a})=\mathrm{T}_{\mathrm{e} 0}(\mathrm{r}=\mathrm{a})$ and $\mathrm{T}_{\mathrm{e}}^{\prime}(\mathrm{r}=0)=0$, where the prime denotes $d / d r$. The normalization scheme is as followings: the length is normalized to a, the magnetic field to $\mathrm{B}_{0 \mathrm{t}}$, and $\mathrm{T}_{\mathrm{e}}$ to $\mathrm{T}_{\mathrm{e} 0}(\mathrm{r}=0)$.

\section{Modelling results}

Equation (4) is solved numerically using the transport subroutines of the initial value code TM1 [9]. TM1 is a new version of our previous code TM for modelling the nonlinear evolution of NTMs and their stabilization by rf wave current drive [30]. In TM1 the new numerical scheme described in Ref. [27] is applied, and both the parallel and the perpendicular transport terms in Eq. (4) are fully implicit.

In the following Part A the heat transport across a single island is studied, and in Part B a two island case is studied. Since the magnetic field becomes stochastic when two islands of different helicity overlap, this allows us to investigate the heat transport across a local stochastic field. A comparison between the numerical and analytical results is given in Part C.

\section{A. Heat transport across a single island}

An example for the change of the radial temperature profile due to a single $m / n=3 / 2$ island is shown in Fig. 1, where the $m / n=0 / 0$ component of $T_{e}, T_{0 / 0}$, and the temperature profile along the line passing through the island's o-point and x-point in the steady state are shown. The original equilibrium temperature $\mathrm{T}_{\mathrm{e} 0}$ is shown by the dotted curve. The $\mathrm{q}=3 / 2$ surface is 
at $r_{3 / 2}=0.604 a$, the island width $w_{3 / 2}=0.0452 \mathrm{a}\left(\psi_{3 / 2,0}=9 \times 10^{-4} \mathrm{aB}_{0 t}\right)$, and $\chi_{\|} / \chi_{\perp}=10^{10}$ is taken. It is seen that the local $\mathrm{T}_{\mathrm{e}}$ profile becomes flattened inside the island when viewing along the line passing through the island's o-point due to the fast parallel transport at a large $\chi_{\|} / \chi_{\perp}$. Along the line passing through the island's $\mathrm{x}$-point, $\mathrm{T}_{\mathrm{e}}$ has a finite radial gradient as expected. The radial gradient of $\mathrm{T}_{0 / 0}$ is same as that of $\mathrm{T}_{\mathrm{e} 0}$ for $\mathrm{r}<\mathrm{r}_{-}$, and $\mathrm{T}_{0 / 0}$ is the same as $\mathrm{T}_{\mathrm{e} 0}$ for $\mathrm{r}>\mathrm{r}_{+}$ due to the conservation of the perpendicular heat flux, where $r_{-}=0.582 \mathrm{a}$ and $r_{+}=0.627 \mathrm{a}$ are the inner and outer edges of the island.

Corresponding to Fig. 1, in Fig. 2 the radial profiles of the fundamental ( $\mathrm{m} / \mathrm{n}=3 / 2$ component) and higher harmonics $\left(\mathrm{m} / \mathrm{n}=6 / 4,9 / 6\right.$ and 12/8 components) of $\mathrm{T}_{\mathrm{e}}$ are shown. Higher harmonic temperature perturbations localize in the island region, and their amplitudes decrease for a larger $\mathrm{m}$ or $\mathrm{n}$, as expected from the infinite $\chi_{\|} / \chi_{\perp}$ limit result [16]. For such a high value of $\chi_{\|} / \chi_{\perp}$ the temperature is a constant along the magnetic field line except in a thin layer around the island's separatrix where the parallel heat flux does not vanish.

In Fig. 3 the radial profiles of the original equilibrium temperature $\mathrm{T}_{\mathrm{e} 0}$ (solid curve) and $\mathrm{T}_{0 / 0}$ in steady state are shown for $\chi_{\|} / \chi_{\perp}=10^{12}, 10^{10}, 10^{8}$ and $10^{6}$, with the other parameters being the same as in Fig. 1. In all our results $\chi_{\perp}$ is kept unchanged. It has been shown by analytical theory that, $\mathrm{T}_{0 / 0}$ is approximately the same as $\mathrm{T}_{\mathrm{e} 0}$ as long as the island width $\mathrm{w}$ is much smaller than the critical island width $\mathrm{w}_{\mathrm{c}}=\mathrm{a}\left(\chi_{\perp} / \chi_{\|}\right)^{1 / 4}\left(\varepsilon \mathrm{an} / 8 \mathrm{~L}_{\mathrm{q}}\right)^{-1 / 2}$, where $\mathrm{L}_{\mathrm{q}}=\mathrm{q} / \mathrm{q}^{\prime}$ and $\varepsilon=\mathrm{a} / \mathrm{R}$ [16]. For infinite ratio of $\chi_{\|} / \chi_{\perp}$, the $\mathrm{T}_{\mathrm{e}}$ profile becomes flattened inside the island, and the decrease of $\mathrm{T}_{0 / 0}$ for $\mathrm{r}<\mathrm{r}_{-}$is $\Delta \mathrm{T}_{\mathrm{e}}=\mathrm{T}_{\mathrm{e} 0}-\mathrm{T}_{0 / 0} \approx \mathrm{T}_{\mathrm{e} 0}{ }^{\prime}\left(\mathrm{r}=\mathrm{r}_{\mathrm{s}}\right) \mathrm{w} / 2^{1 / 2}$ [15]. With our input parameters one finds $\mathrm{w}_{\mathrm{c}}=0.63 \times 10^{-2} \mathrm{a}, 2.0 \times 10^{-2} \mathrm{a}$ and $6.3 \times 10^{-2} \mathrm{a}$ for $\chi_{\|} / \chi_{\perp}=10^{10}, 10^{8}$ and $10^{6}$, respectively. Only for $\chi_{\|} / \chi_{\perp}=10^{6}$, the island width is smaller than the critical island width $\left(\mathrm{w}_{3 / 2} / \mathrm{w}_{\mathrm{c}}=0.72\right)$, so that the difference between $\mathrm{T}_{\mathrm{e} 0}$ and $\mathrm{T}_{0 / 0}$ is small. A larger $\chi_{\|} / \chi_{\perp}$ leads to a more flattening $\mathrm{T}_{0 / 0}$ 
profile across the island and therefore a smaller $\mathrm{T}_{0 / 0}$ for $\mathrm{r}<\mathrm{r}_{\text {. }}$. The $\mathrm{T}_{0 / 0}$ for $\chi_{\|} / \chi_{\perp}=10^{12}$ is very close to that for $\chi_{\|} / \chi_{\perp}=10^{10}$, indicating that the further change in $T_{e}$ is small for $w_{3 / 2} / w_{c}>>1$. It is seen from Fig. 3 that for describing the "large island " limit of NTM [16], $\chi_{\|} / \chi_{\perp}=10^{10}$ has to be taken in the numerical calculations [9].

Corresponding to Fig. 3 , the radial profiles of the $m / n=3 / 2$ component of $T_{e}, T_{3 / 2}$, are shown in Fig. 4 for $\chi_{\|} / \chi_{\perp}=10^{10}, 10^{8}$ and $10^{6}$. For low $\chi_{\|} / \chi_{\perp}$, the magnitudes of $\mathrm{T}_{3 / 2}$ and $\mathrm{T}_{3 / 2}$ ' are small in the island region due to $\mathrm{w}_{3 / 2}<\mathrm{w}_{\mathrm{c}}$, corresponding to a small bootstrap current perturbation for the NTM [16]. A higher $\chi_{\|} / \chi_{\perp}, \chi_{\|} / \chi_{\perp}=10^{8}$, leads to larger local magnitudes of $\mathrm{T}_{3 / 2}$ and $\mathrm{T}_{3 / 2}{ }^{\prime}$. For even larger $\chi_{\|} / \chi_{\perp}\left(10^{10}\right)$, the $\mathrm{T}_{3 / 2}$ profile becomes flattened near the rational surface, in agreement with the infinite $\chi_{\|} / \chi_{\perp}$ limit result [16]. Away from the island these $T_{3 / 2}$ profiles become the same, since $\nabla_{\|} T_{e}$ and high harmonics of $T_{e}$ vanish there.

Considering the conservation of the perpendicular heat flux, $\mathrm{q}_{\perp}=-\chi_{\perp} \mathrm{T}_{0}^{\prime}=-\chi_{\mathrm{e}} \mathrm{T}_{0 / 0}{ }^{\prime}$, where $\chi_{\mathrm{e}}$ is the effective radial heat diffusivity in the presence of the island, one can define an normalized effective radial heat conductivity

$$
\chi=\chi_{\mathrm{e}} / \chi_{\perp} \equiv \mathrm{T}_{\mathrm{e} 0}{ }^{\prime} / \mathrm{T}_{0 / 0}^{\prime}
$$

The enhanced radial heat diffusivity due to the parallel transport across the island is then given by $\chi_{\mathrm{r}}=\chi_{\mathrm{e}}-\chi_{\perp}=\chi_{\perp}(\chi-1)$. Corresponding to Fig. 3, in Fig. 5 radial profiles of $\chi$ are shown for $\chi_{\|} / \chi_{\perp}=10^{10}, 10^{8}$ and $10^{6}$. As expected from Fig. 3, $\chi \approx 1$ for $\chi_{\|} / \chi_{\perp}=10^{6}$, and a larger $\chi_{\|} / \chi_{\perp}$ leads to a larger $\chi$. Along the minor radius $\chi$ has a width being about the same as the island width and a maximal value at the rational surface.

The parameter $\chi_{\mathrm{I}} / \chi_{\perp}=(\chi-1)$ measures the normalized (to $\left.\chi_{\perp}\right)$ enhanced radial heat diffusivity due to the parallel transport. In Fig. 6 the maximal value of $\log (\chi-1)$ at the rational surface (see Fig. 5), $\log (\chi-1)_{\max }$, is shown as a function of $\log \left(\chi_{\|} / \chi_{\perp}\right)$ for the $3 / 2$ island by the solid 
curve, with other parameters being the same as those of Fig. 1 . The condition $w_{3 / 2}=w_{c, 3 / 2}$ leads to $\log \left(\chi_{\|} / \chi_{\perp}\right)_{c}=6.6$, where $\mathrm{w}_{\mathrm{c}, 3 / 2}$ is the critical island width of the $3 / 2$ island. It is seen that, $(\chi-1)_{\max }$ is proportional to $\chi_{\|} / \chi_{\perp}$ for $\chi_{\|} / \chi_{\perp}<\left(\chi_{\|} / \chi_{\perp}\right)_{c}$, since in this case only the quasi-linear correction of $\mathrm{T}_{0 / 0}$ is important when $\mathrm{w}<\mathrm{w}_{\mathrm{c}}$, as shown by the analysis in the Appendix. For $\chi_{\|} / \chi_{\perp}>>\left(\chi_{\|} / \chi_{\perp}\right)_{c}$, however, $(\chi-1)_{\max }$ is proportional to $\left(\chi_{\|} / \chi_{\perp}\right)^{1 / 2}$. Between these two limits there is a transition region around $\sim 10\left(\chi_{\|} / \chi_{\perp}\right)_{\mathrm{c}}$ or $\mathrm{w}_{3 / 2} \approx 1.8 \mathrm{w}_{\mathrm{c}, 3 / 2}$. The doted curve with empty circles in Fig. 6 shows $\log (\chi-1)_{\max }$ for a $m / n=4 / 3$ island, which is quite similar to that of the $3 / 2$ island. The $4 / 3$ island width $\mathrm{w}_{4 / 3}=0.0450 \mathrm{a}$, being approximately the same as $\mathrm{w}_{3 / 2}$, but its critical island width $\mathrm{w}_{\mathrm{c}, 4 / 3}$ is smaller due to its higher toroidal mode number. The condition $\mathrm{w}_{4 / 3}=\mathrm{w}_{\mathrm{c}, 4 / 3}$ leads to $\log \left(\chi_{\|} / \chi_{\perp}\right)_{\mathrm{c}}=6.2$. The larger value of $\log (\chi-1)_{\max }$ for the $4 / 3$ island is due to its smaller $\mathrm{w}_{\mathrm{c}, 4 / 3}$.

In Fig. 7 numerical results of $\log (\chi-1)_{\max }$ versus $\log \left(\mathrm{w} / \mathrm{w}_{\mathrm{c}}\right)^{2}$ for the $3 / 2$ island is shown by the solid curve with circles, and the dotted curve shows the analytical result given by Eq. (A12) in the Appendix. Here in $\left(\mathrm{w} / \mathrm{w}_{\mathrm{c}}\right)^{2}$ only the island width $\mathrm{w}$ changes, $\chi_{\|} / \chi_{\perp}=10^{10}$ and other parameters are the same as those of Fig. 1. It is seen that $(\chi-1)_{\max }$ is proportional to $\mathrm{w}^{4}$ for $w / w_{c}<1$ but to $w^{2}$ for $w / w_{c}>3$. The transition region between these two slopes is around $\mathrm{w} / \mathrm{w}_{\mathrm{c}} \approx 1.8$. When $\mathrm{w} / \mathrm{w}_{\mathrm{c}}<1$, the numerical result is the same as the analytical one given by Eq. (A12). When $w / w_{c}>>1$, the parallel heat transport is along a thin layer around the island's separatrix. The radial layer width is $\mathrm{w}_{\mathrm{c}}{ }^{2} / \mathrm{w}$ at the helical angle being the same as island's o-point [16], where $\mathrm{T}_{\mathrm{e}}{ }_{\mathrm{e}} \approx \mathrm{T}_{\mathrm{e} 0}{ }^{\prime}$. Due to the island's geometry, the layer width expands to $\mathrm{w}_{\mathrm{c}}$ along the minor radius and to $\Delta \zeta \sim\left(4 \mathrm{w}_{\mathrm{c}} / \mathrm{w}\right)$ along the helical angle around island's $\mathrm{x}$-point. Therefore, the $\mathrm{T}_{\mathrm{e}}^{\prime}$ at the $\mathrm{x}$-point $\mathrm{T}_{\mathrm{ex}}{ }^{\prime} \sim\left(\mathrm{w}_{\mathrm{c}} / \mathrm{w}\right) \mathrm{T}_{\mathrm{e} 0}{ }^{\prime}$. Since $\mathrm{T}_{\mathrm{e}}^{\prime}$ is zero well inside the island for $\mathrm{w} / \mathrm{w}_{\mathrm{c}}>>1, \quad \mathrm{~T}_{0 / 0}^{\prime}$, the averaged $\mathrm{T}_{\mathrm{e}}^{\prime}$ along the helical angle, is proportional to 
$\mathrm{T}_{\mathrm{ex}}{ }^{\prime} \Delta \zeta / \pi \sim\left(\mathrm{w}_{\mathrm{c}} / \mathrm{w}\right)^{2} \mathrm{~T}_{\mathrm{e} 0}{ }^{\prime}$. This explains why $(\chi-1)_{\max }$ is proportional to $\left(\chi_{\|} / \chi_{\perp}\right)^{1 / 2}$ and $\mathrm{w}^{2}$ in the limit $\left(w / w_{c}\right)^{2}>>1$, as shown in Figs. 6 and 7.

The degradation of the plasma energy confinement due to an island can be described by the parameter $\Delta \beta=\left(\beta_{0}-\beta\right)$, where $\beta=2 \pi \mathrm{Rn}_{\mathrm{e}} \int 2 \pi \mathrm{r} \mathrm{T}_{0 / 0} \mathrm{dr}$ is the total plasma thermal energy in the presence of the island, and $\beta_{0}=2 \pi R n_{\mathrm{e}} \int 2 \pi \mathrm{rT}$ e0 $\mathrm{dr}$ is that without the island. In Fig. $8 \Delta \beta / \beta_{0}$ is shown as a function of $\log \left(\chi_{\|} / \chi_{\perp}\right)$ for the $3 / 2$ island by the solid curve, with other parameters being the same as those for Fig. 1 . It is seen that $\Delta \beta$ significantly increases with $\log \left(\chi_{\|} / \chi_{\perp}\right)$ from $\log \left(\chi_{\|} / \chi_{\perp}\right)_{c}=6.6$ up to 10 (corresponding $w / w_{c}=1$ to 7.2 ). For $\chi_{\|} / \chi_{\perp}>10^{10}, \Delta \beta$ essentially saturates. When $\mathrm{w} / \mathrm{w}_{\mathrm{c}}>>1, \mathrm{~T}_{\mathrm{e}}$ only slightly changes around the island separatrix, and $\Delta \beta$ is mainly determined by the $\Delta \mathrm{T}_{\mathrm{e}}$ for $\mathrm{r}<\mathrm{r}_{\text {- }}$ as seen from Fig. 3 . Therefore, there is no significant increase of $\Delta \beta$ with $\chi_{\|} / \chi_{\perp}$ for $\chi_{\|} \chi_{\perp}>10^{10}$. Using the analytical formula for the $\chi_{\|} / \chi_{\perp}=\infty$ limit [15], $\Delta \beta / \beta_{0}=0.041$ is obtained, in agreement with the numerical result. The $\Delta \beta / \beta_{0}$ due to the $4 / 3$ island is also shown in Fig. 8 by the dotted curve. The $q=4 / 3$ surface is at $r_{4 / 3}=0.569 a$ with $r_{4 / 3}<r_{3 / 2}$, and all the other parameters are the same as mentioned for Fig. 6. For small $\chi_{\|} / \chi_{\perp}$, the $\Delta \beta / \beta_{0}$ due to the $4 / 3$ island is larger than that due to the $3 / 2$ island, since the temperature flattening inside the $4 / 3$ island occurs at a smaller $\chi_{\|} / \chi_{\perp}$ due to its smaller $\mathrm{w}_{\mathrm{c}}$. For large $\chi_{\|} / \chi_{\perp}$, the $\Delta \beta / \beta_{0}$ due to the $3 / 2$ island is larger because $r_{3 / 2}>r_{4 / 3}$ and $w>>w_{c}[15]$.

\section{B. Heat transport across two islands and stochastic field}

In this part the heat transport across two islands, the $m / n=3 / 2$ and $4 / 3$ islands, is studied. The corresponding rational surfaces are at $r_{3 / 2}=0.604 a$ and $r_{4 / 3}=0.569 a$, respectively. When these two islands are large enough to overlap, the local magnetic field becomes stochastic, allowing us to study the heat transport across a local stochastic field. 
In Fig. 9 an example of the local magnetic surface on the $r-\theta$ plan at $\phi=0$ is shown for $\psi_{0} \equiv \psi_{\mathrm{i}, 0} / \mathrm{aB}_{0 \mathrm{t}}=\psi_{3 / 2,0} / \mathrm{aB}_{0 \mathrm{t}}=\psi_{4 / 3,0} / \mathrm{aB}_{0 \mathrm{t}}=10^{-4}$. In this case the $\mathrm{m} / \mathrm{n}=3 / 2$ and $4 / 3$ islands are not large enough to overlap. Nevertheless, an additional secondary $\mathrm{m} / \mathrm{n}=7 / 5$ island exists due to the coupling between the $3 / 2$ and the $4 / 3$ magnetic field perturbations.

Corresponding to Fig. 9, the local temperature contour is shown in Fig. 10 for $\chi_{\|} / \chi_{\perp}=10^{12}$. In addition to $m / n=3 / 2$ and $4 / 3$ islands, there is also a $m / n=7 / 5$ island in the temperature contour in agreement with Fig. 9. In this case $P(r)=P_{0}(r / a)^{8}\left[1-(r / a)^{2}\right]^{8}$ is taken to deposit more power density in the island region for viewing the $7 / 5$ island more clearly. The small $7 / 5$ island can be seen in the temperature contour only for $\chi_{\|} \chi_{\perp}>10^{10}$, indicating that a small secondary island is important in the transport only for a sufficiently large $\chi_{\|} / \chi_{\perp}$.

With increasing perturbation amplitude $\psi_{0}$, more secondary islands are seen and the magnetic surface first becomes stochastic around the island's separatrix. For sufficiently large $\psi_{0}$, the local magnetic field becomes stochastic. Fig. 11 shows such an example for $\psi_{0}=9 \times 10^{-4}$, leading to $\mathrm{w}_{3 / 2}=0.0452 \mathrm{a}, \mathrm{w}_{4 / 3}=0.0450 \mathrm{a}$ and $\left(\mathrm{w}_{3 / 2}+\mathrm{w}_{4 / 3}\right) /\left(2\left|\mathrm{r}_{3 / 2}-\mathrm{r}_{4 / 3}\right|\right)=1.29$, where $\mathrm{w}_{3 / 2}$ and $\mathrm{w}_{4 / 3}$ are calculated by the conventional single island formula [2].

Corresponding to Fig. 11, in Fig. 12 the local radial profiles of $\mathrm{T}_{\mathrm{e} 0}$ (dot-dashed curve) and the $\mathrm{T}_{0 / 0}$ for $\chi_{\|} / \chi_{\perp}=10^{10}, 10^{8}$ and $10^{6}$ are shown. For low $\chi_{\|} / \chi_{\perp}, \mathrm{T}_{0 / 0}$ is close to $\mathrm{T}_{\mathrm{e} 0}$ similar to a single island. A large $\chi_{\|} / \chi_{\perp}$ leads to a flattening $\mathrm{T}_{0 / 0}$ profile across the island region.

Corresponding to Fig. 12, in Fig. 13 radial profiles of $\log (\chi-1)$ are shown for $\chi_{\|} \chi_{\perp}=10^{10}$, $10^{9}$ and $10^{8}$. For $\chi_{\|} / \chi_{\perp}=10^{8}, \chi$ has only two peaks around the $\mathrm{q}=3 / 2$ and $4 / 3$ surfaces, and its magnitude is approximately the same as that of a single island as shown in Fig. 5. For $\chi_{\|} \chi_{\perp}=10^{10}$ additional peaks of $\chi$ due to the ergodicity are apparent. Comparing with Fig. 5 it 
is found that, $\chi$ is about 5 times larger than that due to a single island with $\chi_{\|} / \chi_{\perp}=10^{10}$, indicating that the ergodicity leads to fast radial transport for large $\chi_{\|} / \chi_{\perp}$.

In Fig. 14 the radial profiles of $\mathrm{T}_{\mathrm{e} 0}$ and the $\mathrm{T}_{0 / 0}$ for $\psi_{0}=2 \times 10^{-4}, 5 \times 10^{-4}$ and $9 \times 10^{-4}$ are shown with $\chi_{\|} / \chi_{\perp}=10^{10}$. For $\psi_{0}=2 \times 10^{-4}, \mathrm{~T}_{0 / 0}$ is approximately the same as $\mathrm{T}_{\mathrm{e} 0}$ between the two island, indicating that the local confinement is not destroyed, although there are secondary islands similar to that shown in Fig. 9. With the increase of the island width, the two islands overlap, and the local $\mathrm{T}_{0 / 0}^{\prime}$ decreases. When plotting the magnetic surface with $\psi_{0}=5 \times 10^{-4}$, it is found to be stochastic in the region $\mathrm{r}=0.58 \mathrm{a}-0.59 \mathrm{a}$ similar to Fig. 11 . However, $\mathrm{T}_{0 / 0}$ is larger there than that at the two rational surfaces.

To study the difference in energy confinement between the cases with and without ergodicity, in Fig. $15 \Delta \beta / \beta_{0}$ is shown as a function of $\log \left(\chi_{\|} / \chi_{\perp}\right)$ by the solid curve for $\psi_{0}=9 \times 10^{-4}$. The corresponding magnetic surface is shown in Fig. 11. The dotted curved shows the $\Delta \beta_{1} / \beta_{0}=\left(\Delta \beta_{3 / 2}+\Delta \beta_{4 / 3}\right) / \beta_{0}$, where $\Delta \beta_{3 / 2}\left(\Delta \beta_{4 / 3}\right)$ is the $\Delta \beta$ due to a single $m / n=3 / 2$ (4/3) island, as shown in Fig. 8. The difference between $\Delta \beta$ and $\Delta \beta_{1}$ therefore measures the additional degradation in the energy confinement due to the ergodicity of the magnetic field. It is seen that $\Delta \beta \approx \Delta \beta_{1}$ for $\chi_{\|} / \chi_{\perp}<3 \times 10^{7}$, corresponding to $\mathrm{w}_{3 / 2}<1.7 \mathrm{w}_{3 / 2, \mathrm{c}}$. This means that for a sufficiently low $\chi_{\|} / \chi_{\perp}$, the ergodicity does not affect the energy confinement, and the heat transport recognizes the individual island structure rather than the ergodicity. For $\chi_{\|} / \chi_{\perp}>3 \times 10^{7}, \Delta \beta$ becomes larger than $\Delta \beta_{1}$, showing the additional degradation of the confinement due to ergodicity. However, such an additional degradation is not significant with $\chi_{\|} / \chi_{\perp}$ up to $10^{11}$, which is of the same order of magnitude as that for the central region plasma of large tokamaks [16]. The small difference between $\Delta \beta$ and $\Delta \beta_{1}$ is due to the fact that, $\Delta \beta$ is mainly determined by 
the $\Delta \mathrm{T}_{\mathrm{e}}$ for $\mathrm{r}<\mathrm{r}_{\text {- }}$ of the inner island, the $4 / 3$ island in the present case, and $\Delta \mathrm{T}_{\mathrm{e}}$ changes little for sufficiently large $\chi_{\|} / \chi_{\perp}$ similar to that of a single island as seen from Fig. 3 .

In Fig. $16 \log (\chi-1)$ versus $\log \left(\chi_{\|} / \chi_{\perp}\right)$ is shown for $\psi_{0}=9 \times 10^{-4}$ by the solid curve. The value of $\chi$ is taken at $r=0.587 \mathrm{a}$ where it has a local minimum value (see Fig. 13). One finds $(\chi-1) \propto \chi_{\|} / \chi_{\perp}$ in the low $\chi_{\|} / \chi_{\perp}$ limit in agreement with Eq. (A14) in the Apendix. For $\chi_{\|} / \chi_{\perp}>3 \times 10^{8}\left(\mathrm{w}_{3 / 2}>3.2 \mathrm{w}_{\mathrm{c}, 3 / 2}\right),(\chi-1)$ again approximately scales as $\chi_{\|} / \chi_{\perp}$. Between these two limits there is a transition region around $\chi_{\|} / \chi_{\perp}=3 \times 10^{7}\left(\mathrm{w}_{3 / 2}=1.7 \mathrm{w}_{\mathrm{c}, 3 / 2}\right)$. In this region $(\chi-1)$ increases less than linear with $\chi_{\|} \chi_{\perp}$, similar to the case of a single island with $\mathrm{w}_{\sim} \mathrm{w}_{\mathrm{c}}$. The dotted curve in Fig. 16 shows $\log \left(\chi_{3 / 2}+\chi_{4 / 3}-2\right)$, where $\chi_{3 / 2}\left(\chi_{4 / 3}\right)$ is the $\chi$ obtained with a single $3 / 2(4 / 3)$ island at the same radial location. The solid curve is approximately the same as the dotted one for $\chi_{\|} / \chi_{\perp}<3 \times 10^{7}$, as predicted by Eq. (A14) that the heat transport is determined by the additive effects of the individual islands for $\mathrm{w}<\mathrm{w}_{\mathrm{c}}$. When plotting the radial profiles for the case $\mathrm{w}<<\mathrm{w}_{\mathrm{c}}, \log (\chi-1)$ is found to be the same as $\log \left(\chi_{3 / 2}+\chi_{4 / 3}-2\right)$ everywhere across the island region. For sufficiently large $\chi_{\|} / \chi_{\perp}$, the solid curve is larger than the dotted one, indicating the role of the stochastic field.

With an even larger magnetic field perturbation amplitude, $\psi_{0}=3.6 \times 10^{-3}$, the local magnetic surface becomes fully ergodic, and the results are found to be similar to those shown in Fig. 16. In addition to the case of overlapping the $m / n=3 / 2$ and $4 / 3$ islands discussed above, studies have also been carried out for the $m / n=30 / 20$ and $30 / 21$ islands. In this case the local magnetic field is more stochastic due to a shorter distance between the two rational surfaces, but results similar to those shown in Figs. 15 and 16 have been found.

It is seen from the results above that, similar to the single island case, the parameter $\mathrm{w} / \mathrm{w}_{\mathrm{c}}$ also characterizes the heat diffusion across a local stochastic field. For $w<<w_{c}$, the heat 
transport is determined by the additive effects of the individual islands, and $\chi_{\mathrm{r}} \propto \chi_{\|}$is found as predicted by the analytical result Eq. (A14). Around $\mathrm{w}=1.7 \mathrm{w}_{\mathrm{c}}$ there is a transition region where the additive effect of individual islands is still important, and $\chi_{\mathrm{r}}$ only slowly increases with $\chi_{\|}$. For sufficiently large $\mathrm{w} / \mathrm{w}_{\mathrm{c}}, \mathrm{w} / \mathrm{w}_{\mathrm{c}}>3, \chi_{\mathrm{r}}$ nearly scales with $\chi_{\|}$, but the heat diffusion is dominated by the ergodicity of the magnetic field.

\section{Comparison with analytical theories}

There have been extensive theoretical studies on the heat transport across the stochastic field due to the spacial diffusion of the magnetic field lines [17-24]. In the collisionless regime where the electron mean free path $\lambda_{\mathrm{e}}$ is longer than the Kolmogorov length $\mathrm{L}_{\mathrm{k}} \approx\left[\mathrm{L}_{\mathrm{s}}^{2} /\left(\mathrm{k}_{\perp}^{2} \mathrm{D}_{\mathrm{M}}\right)\right]^{1 / 3}[17,20]$, the enhanced radial heat conductivity is shown by Rechester and Rosenbluth to be [17]

$$
\chi_{\mathrm{r}}=\mathrm{D}_{M} \chi_{\|} / \lambda_{e}=\mathrm{D}_{M} \mathrm{v}_{\mathrm{Te}},
$$

where $\mathrm{v}_{\mathrm{Te}}$ is the electron thermal velocity,

$$
\mathrm{D}_{\mathrm{M}}=L_{0} \sum_{k}\left(\frac{b_{k, r}}{B_{0 t}}\right)^{2} \delta\left(m_{k} / q-n_{k}\right),
$$

$\mathrm{L}_{0} \approx \pi \mathrm{R}, \mathrm{b}_{\mathrm{k}, \mathrm{r}}$ is the radial magnetic field perturbation of the $k$ th component, $\mathrm{k}_{\perp}$ is the perpendicular wave vector of the perturbations, $\mathrm{L}_{\mathrm{s}}=\mathrm{Rq}^{2} / \mathrm{rq}^{\prime}$, and the summation is over $\mathrm{k}$ to include contributions from all components. Stix has got the same results as Eq. (7) [18]. In the collisional regime $\lambda_{\mathrm{e}}<\mathrm{L}_{\mathrm{k}}$,

$$
\chi_{\mathrm{r}}=\mathrm{D}_{\mathrm{M}} \chi_{\|} / L_{c \delta}
$$


where $\mathrm{L}_{\mathrm{c} \delta}=\mathrm{L}_{\mathrm{c}} \ln \left[\left(\mathrm{r} / \mathrm{mL}_{\mathrm{c}}\right)\left(\chi_{\|} / \chi_{\perp}\right)^{1 / 2}\right], \mathrm{L}_{\mathrm{c}}=\pi \mathrm{R} / \ln (\pi \Delta / 2), \Delta=\left(\mathrm{w}_{1}+\mathrm{w}_{2}\right) /\left(2\left|\mathrm{r}_{1}-\mathrm{r}_{2}\right|\right), \mathrm{w}_{1}$ and $\mathrm{w}_{2}$ are the widths of two neighboring islands, and $r_{1}$ and $r_{2}$ are the minor radius of the corresponding rational surfaces [17].

Using a fluid approach, Kadomsev and Pogutse calculated the heat transport in several regimes [19], but in no regime was the result the same as the collisional result of Rechester and Rosenbluth [17]. Later Krommes et al showed that the collisional regime consists of three sub-regimes [20]. With the decreasing of $\mathrm{L}_{\lambda}$, they are

(a) Rechester-Rosenbluth regime:

$$
\chi_{r}^{R R}=\mathrm{D}_{\mathrm{M}} \chi_{\|} / L_{k}
$$

being valid for $\tau_{\|}<\tau_{\mathrm{k}}<\tau_{\perp}$, where $\tau_{\|}=\mathrm{L}_{0}^{2} / \chi_{\|}, \tau_{\mathrm{k}}=\mathrm{L}_{\mathrm{k}}^{2} / \chi_{\|}$and $\tau_{\perp}=1 /\left(\mathrm{k}_{\perp}^{2} \chi_{\perp}\right)$. Eq. (10) differs from Eq. (9) by a factor $\mathrm{L}_{\mathrm{c \delta}} / \mathrm{L}_{\mathrm{k}}$.

(b) Kadomsev-Pogutse regime:

$$
\chi_{r}^{K P}=\mathrm{D}_{\mathrm{M}}\left(\chi_{\|} \chi_{\perp}\right)^{1 / 2} \mathrm{k}_{\perp}
$$

being valid for $\tau_{\|}<\tau_{\perp}<\tau_{\mathrm{k}}$.

(c) Fluid regime:

$$
\chi_{r}^{F}=\mathrm{D}_{\mathrm{M}} \chi_{\|} / L_{0},
$$

being valid for $\tau_{\perp}<\tau_{\|}<\tau_{\mathrm{k}}$.

The validities of various regimes depend on electron collisionality and the level of the stochastic magnetic field fluctuations [20,25,26]. The original theory of Krommes et al is even more complicated [20].

Since the fluid equation, Eq. (4), is utilized in our model, it is of interest to have a comparison between our numerical results and the analytical ones in the collisional regime, Eqs. (9)-(12). As shown in the Appendix, when each individual island width, $\mathrm{w}_{\mathrm{k}}$, is smaller than its corresponding critical island width, $\mathrm{w}_{\mathrm{c}, \mathrm{k}}$, the enhanced radial heat diffusivity is given by 
Eq. (A14) rather than Eq. (12), the Fluid regime result. In this case only these magnetic field perturbations with their rational surfaces being sufficiently close to the observation point $r$ have a significant contribution to $\chi_{\mathrm{r}}$, and the heat diffusion is essentially determined by the additive effects of these individual islands satisfying $\left(\mathrm{r}-\mathrm{r}_{\mathrm{s}, \mathrm{k}}\right) / \mathrm{w}_{\mathrm{c}, \mathrm{k}}<2$. The valid regime of Eq. (A14), $\mathrm{w}_{\mathrm{k}} / \mathrm{w}_{\mathrm{c}, \mathrm{k}}<<1$, is also much wider than that of Eq. (12). It is seen from Fig. 16 that, $\chi_{\mathrm{r}}$ is proportional to $\chi_{\|}$for low values of $\chi_{\|} / \chi_{\perp}$ and is determined by the additive effects of these individual islands up to $\mathrm{w}_{3 / 2} / \mathrm{w}_{\mathrm{c}, 3 / 2}<1.7$, as predicted by Eq. (A14).

In the transition region around $\mathrm{w}_{3 / 2} \sim \mathrm{w}_{\mathrm{c}, 3 / 2},(\chi-1)$ slowly increases with $\chi_{\|} / \chi_{\perp}$ as shown by Fig. 16. It is clear that this is due to $\mathrm{w}_{\mathrm{k}} \sim \mathrm{w}_{\mathrm{c}, \mathrm{k}}$ so that the single island effect is still important. The Kadomsev-Pogutse regime given by Eq. (11) is not found from numerical results. In fact, only for a single island with $\mathrm{w}>>\mathrm{w}_{\mathrm{c}}$ one finds $\chi_{\mathrm{r}, \max } \sim\left(\chi_{\|} \chi_{\perp}\right)^{1 / 2}$ (see Fig. 6).

For sufficiently large $\chi_{\|} / \chi_{\perp},(\chi-1)$ given by Fig. 16 approximately scales with $\chi_{\|} / \chi_{\perp}$, suggesting a behavior predicted by Eq. (10).

For a detail comparison with Eqs. (9)-(12), in Fig. $17 \kappa \equiv \mathrm{a}<\chi_{\mathrm{r}}>/\left[\chi_{\|} \mathrm{L}_{0} \sum\left(\mathrm{b}_{\mathrm{k}, \mathrm{r}} / \mathrm{B}_{0 \mathrm{t}}\right)^{2}\right]$ versus $\log \left(\chi_{\|} \chi_{\perp}\right)$ is shown for $\psi_{0}=1 \times 10^{-4}, 6 \times 10^{-4}, 9 \times 10^{-4}, 2.1 \times 10^{-3}$, and $3.0 \times 10^{-3}$, with other parameters being the same as those for Fig. 16. Here $\left\langle\chi_{r}>=\int \chi_{r} d r /\left(r_{b}-r_{a}\right)\right.$ is the radial averaged $\chi_{r}$ from $r_{a}=0.58 a$ to $r_{b}=0.59 a$ where the magnetic field is stochastic (see Fig. 11). It is seen from Fig. 17 that, for low $\chi_{\|} / \chi_{\perp}, \kappa$ is the same for different $\psi_{0}$, as predicted by Eq. (A14) in the limit $\mathrm{z}_{\mathrm{k}}=0$. In this limit $\mathrm{\kappa}=0.048$ is obtained from Eq. (A14) in agreement with the numerical results. For high $\chi_{\|} / \chi_{\perp}$, the value of $\kappa$ oscillates for $\psi_{0}<2 \times 10^{-3}$ but approaches a nearly steady value for lager $\psi_{0}$. This differs from the prediction of Eq. (10) that $\kappa \sim 1 / L_{k} \sim D_{M}^{1 / 3} \sim \psi_{0}^{2 / 3}$. Using Eq. (10), one finds $\kappa=9.28 \times 10^{-3}$ for $\psi_{0}=3 \times 10^{-3}$. 
By increasing the magnetic shear by 3 times to $L_{q}=0.1 \mathrm{a}$, in Fig. $18 \kappa$ versus $\log \left(\chi_{\|} / \chi_{\perp}\right)$ is shown for $\psi_{0}=3.0 \times 10^{-3}, 4.5 \times 10^{-3}$ and $6.0 \times 10^{-3}$. In this case $r_{3 / 2}=0.595 \mathrm{a}, \mathrm{r}_{4 / 3}=0.583 \mathrm{a}$ and the other parameters are the same as that for Fig. 17. It is seen that $\kappa$ approaches a steady value for low $\chi_{\|} / \chi_{\perp}$ and a nearly steady value for high $\chi_{\|} / \chi_{\perp}$ and $\psi_{0}$, similar to those of Fig. 17. The dotted curve on Fig. 18 is the result with a smaller magnetic shear, $\mathrm{L}_{\mathrm{q}}=0.3 \mathrm{a}$, and $\psi_{0}=3.0 \times 10^{-3}$, shown here for comparison. $\kappa$ becomes smaller for a larger magnetic shear, differing from the prediction of Eq. (10) that $\kappa \sim 1 / \mathrm{L}_{\mathrm{k}} \sim \mathrm{L}_{\mathrm{s}}^{-2 / 3}$. The dashed curve on Fig. 18 shows the result of Eq. (9) for $\psi_{0}=6.0 \times 10^{-3}$ and $\mathrm{L}_{\mathrm{q}}=0.1 \mathrm{a}$, which is different from the numerical results.

Numerical calculations have also been carried out by using magnetic perturbations with four Fourier components, $\mathrm{m} / \mathrm{n}=30 / 19,30 / 20,30 / 21$, and 30/22, and five components, $\mathrm{m} / \mathrm{n}=3 / 2,4 / 3,7 / 5,10 / 7$, and $11 / 8$. In these cases the local field is more stochastic due to shorter distance between rational surfaces, and similar results to Figs. 17 and 18 are found. An example of four island case is shown in Fig. 19. In this case the rational surfaces are at $r_{30 / 19}=0.620 a, r_{30 / 20}=0.604 a, r_{30 / 21}=0.595 a$, and $r_{30 / 22}=0.576 a$, and the radial average is taken from $\mathrm{r}=0.59 \mathrm{a}$ to $0.61 \mathrm{a}$ where the magnetic field is stochastic. $\kappa$ is shown for $\psi_{0}=9.0 \times 10^{-4}$, $1.2 \times 10^{-3}, 1.8 \times 10^{-3}$ and $2.1 \times 10^{-3}$. It is seen that $\kappa$ approaches a steady value for low $\chi_{\|} / \chi_{\perp}$ and a nearly steady value for high $\chi_{\|} \chi_{\perp}$ and $\psi_{0}$, similar to those of Fig. 17 . The dotted curve on Fig. 19 is the result with only the $3 / 2$ and $4 / 3$ island and $\psi_{0}=2.1 \times 10^{-3}$, shown here for comparison. The $\kappa$ for the four island case is smaller due to higher mode numbers and therefore smaller critical island widths.

It is seen from Figs. 16-19 that, the quasi-linear result presented in the Appendix is confirmed by numerical results, while the Kadomsev-Pogutse regime is not seen. As for the Rechester-Rosenbluth regime given by Eq. (10), the scaling $\chi_{\mathrm{r}}^{\mathrm{RR}} \sim \chi_{\|}$is found for a sufficiently 
large $\chi_{\|} / \chi_{\perp}$ from numerical results, but the scaling with the magnetic shear and the perturbation amplitude is different.

\section{Discussion and summary}

It is clear from above results that, $\mathrm{w} / \mathrm{w}_{\mathrm{c}}$ is a key parameter in determining the heat transport across a single island. The heat diffusion consists of three regimes: (a) the quasi-linear regime $\mathrm{w} / \mathrm{w}_{\mathrm{c}}<1,(\mathrm{~b})$ the transitional regime $\mathrm{w} \sim \mathrm{w}_{\mathrm{c}}$ and (c) the "large island" regime $\mathrm{w}>>\mathrm{w}_{\mathrm{c}}$. The energy confinement degradation significantly increases with $\mathrm{w} / \mathrm{w}_{\mathrm{c}}$ from $\mathrm{w} / \mathrm{w}_{\mathrm{c}}=1$ to 7.2 . For $\mathrm{w} / \mathrm{w}_{\mathrm{c}}>7.2$, the degradation is determined by $\mathrm{w}, \mathrm{T}_{\mathrm{e} 0}{ }^{\prime}\left(\mathrm{r}=\mathrm{r}_{\mathrm{s}}\right)$ and $\mathrm{r}_{\mathrm{s}}$ as found in the infinite $\chi_{\|} / \chi_{\perp}$ limit [15]. As for the radial gradient of the fundamental harmonic, it takes its maximal value in the island region in the middle range of $\mathrm{w} / \mathrm{w}_{\mathrm{c}}$, and the "large island" limit of NTM discussed in Ref. [16] is for $w / w_{c}>7.2$.

For the local stochastic magnetic field due to the overlap of two neighboring islands, the parameter $\mathrm{w} / \mathrm{w}_{\mathrm{c}}$ is also found to be important in characterizing the transport. In different to the Ref. [20], we find that the heat diffusion consists of three regimes:

(a) The quasi-linear regime $\mathrm{w} / \mathrm{w}_{\mathrm{c}}<1$ as shown by Eq. (A14). In this regime the transport is determined by the additive effect of individual islands.

(b) The transitional regime $\mathrm{w} \sim \mathrm{w}_{\mathrm{c}}$, where $\chi_{\mathrm{r}}$ slowly increases with $\chi_{\|}$.

(c) The regime $\mathrm{w} \gg>\mathrm{w}_{\mathrm{c}}$, where $\chi_{\mathrm{r}}$ approximately scales with $\chi_{\|}$.

Our numerical results together with the analysis in the Appendix indicate that, the Fluid regime should be replaced by the quasi-linear results Eq. (A14). The Kadomsev-Pogutse regime is not found from the numerical results as expected from Eq. (A14). It is seen from our results that, the effect of $\mathrm{w} / \mathrm{w}_{\mathrm{c}}$ not considered in previous analytical theories is important in determining the transport for $\mathrm{w} / \mathrm{w}_{\mathrm{c}} \leq 1$ and leads to the difference between our numerical 
results and Eqs. (9), (11) and (12). Recent studies have shown that, the magnetic field shear plays an important in the spacial diffusion of the field lines, and the excursions of field lines significantly differ from Brownian motions [24].

It should be mentioned that, there is a difference between our numerical model and that of previously analytical theories [17-20]. Our model is a local stochastic field due to the overlap of two or several islands, while in Ref. [20] a infinite stochastic field is assumed. It is not clear whether such a difference could lead to the difference in the scaling of $\chi_{\mathrm{r}}$ with the magnetic shear and the perturbation amplitude, although $\chi_{\mathrm{r}} \sim \chi_{\|}$is obtained from the numerical results for a sufficiently large perturbation amplitude and $\chi_{\|} / \chi_{\perp}$. Future calculations using a non-local stochastic field and including more Fourier components of magnetic perturbations will be helpful for a further comparison with Eq. (10).

Eq. (A14) have an important implication on the heat diffusion across a stochastic field where $\chi_{\|} / \chi_{\perp}$ is not high enough. For the tokamak edge parameters $T_{e}=100 e v, n_{e}=10^{19} \mathrm{~m}^{-3}$, $\mathrm{L}_{\mathrm{q}}=\mathrm{q} / \mathrm{q}^{\prime}=\mathrm{a}, \mathrm{R} / \mathrm{a}=3, \mathrm{n}=2$, and an anomalous perpendicular heat diffusivity, $\chi_{\perp}=0.5 \mathrm{~m}^{2} / \mathrm{s}$, one finds $\chi_{\|} / \chi_{\perp}=1.7 \times 10^{8}$ and $\mathrm{w}_{\mathrm{c}}=0.03 \mathrm{a}$ by using the classical parallel electron heat conductivity $\chi_{\| \mathrm{c}}=3.16 \mathrm{v}_{\mathrm{Te}} \lambda_{\mathrm{e}}$. This means that for smaller islands $(\mathrm{w}<0.03 \mathrm{a})$ the field ergodicity plays no role, and the heat diffusion is determined by the additive effect of individual islands. Only for sufficiently large islands or high $\mathrm{T}_{\mathrm{e}}$, the ergodicity dominates the radial transport.

The validity of Eq. (4) and the constant $\chi_{\|}$assumption in our calculations should be discussed. It was shown by the analytical theory that, the classical heat conductivity $\chi_{\| \mathrm{lc}}$ is valid for $\mathrm{k}_{\|} \lambda_{\mathrm{e}}<1$. While for $\mathrm{k}_{\|} \lambda_{\mathrm{e}}>1, \chi_{\|} \approx \mathrm{v}_{\mathrm{Te}} / \mathrm{k}_{\|}$, where $\mathrm{k}_{\|}=\mathbf{B}_{0} \cdot \mathbf{k} /\left|\mathbf{B}_{0}\right|$ and $\mathbf{k}$ is the wave vector of the island [28]. In the lowest order $\mathrm{k}_{\|}=\mathrm{n} \mid \mathrm{r}-\mathrm{r}_{\mathrm{s}} / /\left(\mathrm{L}_{\mathrm{q}} \mathrm{R}\right)$, where $\mathrm{r}_{\mathrm{s}}$ is the minor radius of the rational surface. With the parameters mentioned above, $\mathrm{k}_{\| \mid} \lambda_{\mathrm{e}}>1$ leads to $\left|\mathrm{r}-\mathrm{r}_{\mathrm{s}}\right|>0.24 \mathrm{a}$. For an island with its width $\mathrm{w}<0.24 \mathrm{a}$, across the island region $\chi_{\|}$is still by $\chi_{\| \mathrm{c}}$. Since $\mathrm{T}_{\mathrm{e}}$ is essentially a constant 
in the island region, the assumption of a constant $\chi_{\|}$is reasonable. In the outer region away from the island one has $\mathrm{k}_{\|} \lambda_{\mathrm{e}}>1$ and $\chi_{\|} \approx \mathrm{v}_{\mathrm{Te}} / \mathrm{k}_{\|}$. In this region, however, the use of $\chi_{\|} \approx \mathrm{v}_{\mathrm{Te}} / \mathrm{k}_{\|}$or $\chi_{\| \mathrm{c}}$ will lead to the same result: the temperature along the field lines becomes a constant due to the fast parallel transport, since both forms of $\chi_{\|}$are large enough to lead to $\chi_{\|} / \chi_{\perp}>>1$ (see Fig. 4 and Appendix). Therefore, for the above case, being relevant to the tokamak edge plasmas such as those in the dynamic ergodic divertor and the edge stochastic field $[31,32]$, the use of $\chi_{\| \mathrm{c}}$ leads to the correct results both in the island and in the outer region.

For a higher electron temperature and density, $\mathrm{T}_{\mathrm{e}}=1 \mathrm{kev}, \mathrm{n}_{\mathrm{e}}=5 \times 10^{19} \mathrm{~m}^{-3}$, and the other parameters being the same as mentioned above, one finds $\chi_{\| \mathrm{c}} / \chi_{\perp}=1.1 \times 10^{10}$, and $\mathrm{k}_{\|} \lambda_{\mathrm{e}}>1$ leads to $\left|r-r_{s}\right| / a>0.012$. Since the region $k_{\|} \lambda_{e}<1$ is very narrow for a high $T_{e}$ case, a modified form of $\chi_{\|}, \chi_{\|}=\chi_{\| c}\left[1+\left(3.16 \lambda_{\mathrm{e}} \mathrm{k}_{\|}\right)^{2}\right]^{1 / 2}$ was used before [30], which reduces to $\chi_{\| \mathrm{c}}$ in the limit $\mathrm{k}_{\|} \lambda_{\mathrm{e}}<<1$ and to $\mathrm{v}_{\mathrm{Te}} / \mathrm{k}_{\| \mid}$in the opposite limit. However, as mentioned in the Introduction, $\chi_{\|}=\mathrm{v}_{\mathrm{Te}} / \mathrm{k}_{\|}$is only a crude approximation for the $\mathrm{k}_{\|} \lambda_{\mathrm{e}}<<1$ limit [29]. Future calculations with a more exact model for the parallel heat flux is necessary for high ratio of $\chi_{\|} / \chi_{\perp}$.

In our work only the electron heat transport is studied. For the ion heat transport across magnetic islands, the results are expected to be similar. Since the parallel ion heat transport are $\left(\mathrm{m}_{\mathrm{i}} / \mathrm{m}_{\mathrm{e}}\right)^{1 / 2}$ times slower than the electron's, the corresponding $\mathrm{w}_{\mathrm{c}}$ is $\left(\mathrm{m}_{\mathrm{i}} / \mathrm{m}_{\mathrm{e}}\right)^{1 / 8}$ times larger, leading to a factor about 3 for a deuterium plasma. Therefore, a larger island width is required to affect the ion temperature profile.

The heat transport is studied here with given perturbed magnetic fields. Spontaneous growing islands are often observed in tokamak experiments $[3,12,33,34]$. Theoretically, the micro-tearing mode could lead to small islands [10]. It has been shown recently that the drift-tearing mode with high mode numbers can be driven unstable by the electron 
temperature gradient due to the perpendicular heat transport [11]. Further investigation on the nonlinear mode saturation is necessary for calculating their effect on the transport.

In summary, it is found in the present paper that:

(1) The heat transport across a single island is determined the parameter $w / w_{c}$. The normalized enhanced radial heat diffusivity due to the island, $\chi_{\mathrm{r}} / \chi_{\perp}$, has a radial width being about the island width and a maximal value at the rational surface being proportional to $\left(\mathrm{w} / \mathrm{w}_{\mathrm{c}}\right)^{2}$ for $\mathrm{w} / \mathrm{w}_{\mathrm{c}}>3$. While for $\mathrm{w} / \mathrm{w}_{\mathrm{c}}<1, \chi_{\mathrm{r}} / \chi_{\perp} \propto\left(\mathrm{w} / \mathrm{w}_{\mathrm{c}}\right)^{4}$. Between these two limits there is a transition region around $\mathrm{w} / \mathrm{w}_{\mathrm{c}}=1.8$. The energy confinement degradation significantly increases with $\mathrm{w} / \mathrm{w}_{\mathrm{c}}$ from $\mathrm{w} / \mathrm{w}_{\mathrm{c}}=1$ to 7.2 .

(2) The heat transport across a local stochastic magnetic field due to the overlap of two or several neighboring islands is also characterized by $w / w_{c}$. For $w<w_{c}$, the heat transport is determined by the additive effects of the individual islands as predicted by Eq. (A14). Around $\mathrm{w}=1.7 \mathrm{w}_{\mathrm{c}}$ there is a transition region where $\chi_{\mathrm{r}}$ slowly increases with $\chi_{\|}$, and the additive effects of the individual islands is still important. For sufficiently large $w / w_{c}, w / w_{c}>3, \chi_{r}$ approximately scales with $\chi_{\|}$.

(3) The Fluid regime should be replaced by the quasi-linear results given by Eq. (A14), and the Kadomsev-Pogutse regime is not found from the numerical results. As for the RechesterRosenbluth regime, the scaling $\chi_{\mathrm{r}} \sim \chi_{\|}$is obtained from the numerical results for $\mathrm{w}>>\mathrm{w}_{\mathrm{c}}$, but the scaling of $\chi_{\mathrm{r}}$ with the magnetic shear and the perturbation amplitude is different for a local stochastic field. 


\section{Appendix}

In this appendix $\chi_{\mathrm{r}}$ is analyzed in the limit that $\chi_{\|} / \chi_{\perp}>>1$ but $\mathrm{w} / \mathrm{w}_{\mathrm{c}}<<1$. Assuming $\mathrm{n}_{\mathrm{e}}, \chi_{\|}$ and $\chi_{\perp}$ to be constant, in steady state Eq. (4) becomes

$$
\chi_{\|} \nabla \cdot\left(\nabla_{\|} \mathrm{T}_{\mathrm{e}}\right)+\chi_{\perp} \nabla \cdot\left(\nabla_{\perp} \mathrm{T}_{\mathrm{e}}\right)+\mathrm{P}(\mathrm{r}) / \mathrm{n}_{\mathrm{e}}=0
$$

When there is only one Fourier component of magnetic perturbations, $\mathbf{B}_{1}=\nabla \psi_{1} \times \mathbf{e}_{\mathrm{t}}$ with

$$
\psi_{1}(r, \theta, \phi)=\psi_{1}(r) \cos (i \xi),
$$

$\mathrm{T}_{\mathrm{e}}$ can be expressed in terms of Fourier series,

$$
T_{\mathrm{e}}=T_{0 / 0}(r, t)+\sum\left[T_{k}(r, t) \exp (i k \xi)+c . c .\right] / 2,
$$

where $\xi=\mathrm{m} \theta+\mathrm{n} \phi$ being the helical angle, and the summation is over $\mathrm{k}$ from $\mathrm{k}=1$ to infinity.

If the island width $\mathrm{w}$ is much smaller than the critical island width $\mathrm{w}_{\mathrm{c}}$, one can define a small expansion parameter $\delta=\mathrm{w} / \mathrm{w}_{\mathrm{c}}$. Taking the operator $\int \mathrm{d} \zeta \cos \zeta / \pi$ with the integration from $\zeta=0$ to $2 \pi$, it is found from Eqs. (A1)-(A3) that in the lowest order in $\delta$

$$
\chi_{||}\left(-k_{\|}^{2} T_{1}+i k_{\|} b_{1 r} T_{0}^{\prime} / B_{0 t}\right)+\chi_{\perp} \nabla \cdot\left(\nabla_{\perp} T_{1}\right)=0
$$

where $\mathrm{k}_{\|}=\mathbf{k}_{1} \cdot \mathbf{B}_{0} /\left|\mathbf{B}_{0}\right|$, and $\mathbf{k}_{1}$ is the wave vectors of $\mathbf{B}_{1}$. In Eq. (A4) the terms containing $\mathrm{T}_{\mathrm{k}}$ with $\mathrm{k} \geq 2$ are neglected since they are smaller by at least an order $\delta^{2}$.

For $\chi_{\|} \mid \chi_{\perp}>>1$ and away from the rational surface, Eq. (A4) is dominated by the first two terms, and the outer region solution $\mathrm{T}_{1}$ is simply determined by

$$
T_{1}=i T_{0 / 0}^{\prime} b_{1 r} /\left(k_{\|} B_{0 t}\right) \text {. }
$$

In the inner region near the rational surface $r_{s}$ with $\left|r-r_{s}\right|<<r_{s}, T_{1}$ has a large radial derivative since $T_{1} \sim 1 /\left(r-r_{s}\right)$ as $r$ approaches $r_{s}$, as seen from Eq. (A5), so that $T_{1}^{\prime}>>m T_{1} / r$ and Eq. (A4) is simplified to

$$
\left(\chi_{\perp} / \chi_{||}\right) T_{1}^{\prime \prime}-k_{\|}^{2} T_{1}=-i k_{\|} T_{e 0}^{\prime} b_{1 r} / B_{O t} .
$$


The balancing between the two terms on the right hand side of Eq. (A6) leads to the critical width $\mathrm{w}_{\mathrm{c}}$. Eq. (A6) can be written into the form

$$
\frac{d^{2} f}{d z^{2}}-z^{2} f=z
$$

where $\mathrm{z}=2^{3 / 2}\left(\mathrm{r}-\mathrm{r}_{\mathrm{s} 1}\right) / \mathrm{w}_{\mathrm{c}}, \mathrm{T}_{1}=-2^{-5 / 2}\left(\mathrm{w}^{2} / \mathrm{w}_{\mathrm{c}}\right) \mathrm{T}_{\mathrm{s}}{ }^{\prime} \mathrm{f}(\mathrm{z})$, and $\mathrm{T}_{\mathrm{s}}{ }^{\prime}=\mathrm{T}_{\mathrm{e} 0}{ }^{\prime}\left(\mathrm{r}_{\mathrm{s}}\right)$. The solution of Eq. (A7) is

$$
f(z)=-\frac{z}{2} \int_{0}^{1} d \mu\left(1-\mu^{2}\right)^{-1 / 4} \exp \left(-z \mu^{2} / 2\right)
$$

For $z>>1, f(z)=-1 / z$, and $T_{1}$ matches to the outer region solution given by Eq. (A5). While for $\mathrm{z}<<1, \mathrm{f}(\mathrm{z}) \approx-1.20 \mathrm{z}$, and

$$
T_{1}=0.3\left(w / w_{c}\right)^{2} T_{s}^{\prime}\left(r-r_{s}\right),
$$

in agreement with Ref. [16]. It can be shown from Eqs. (A1)-(A3) that $\mathrm{T}_{\mathrm{k}}$ with $\mathrm{k} \geq 2$ are $\delta^{2(\mathrm{k}-1)}$ times smaller than $T_{1}$.

Similar to the procedure for obtaining Eq. (A6), by taking the operator $\int d \zeta / \pi$ with the integration from $\zeta=0$ to $2 \pi$ and keeping the terms in the lowest order, the equation for $T_{0 / 0}$ is found from Eqs. (A1)-(A3) to be

$$
0.5 \chi_{\|} b_{1 r}\left[i k_{\|} T_{1}+b_{1 r} T_{0}^{\prime} / B_{0 t}\right]^{\prime}+\chi_{\perp}\left(T_{0 / 0}-T_{0}\right)^{\prime \prime}=0
$$

The neglected terms in Eq. (A10) are at least $\delta^{2}$ times smaller. Substituting $\mathrm{T}_{1}$ into Eq. (A10), one finds $\delta \mathrm{T}=\mathrm{T}_{0 / 0}-\mathrm{T}_{\mathrm{e} 0}$ to be

$$
\delta T(z)=-\frac{w^{4}}{8 w_{c}^{4}} T_{s} g(z),
$$

where $g(z)=[1+z f(z)]$. The profiles of $f(z)$ and $g(z)$ are shown in Fig. 20. $g(z)$ approaches zero for $\mathrm{z}>2$.

The parameter $\chi_{\mathrm{r}}=\chi_{\perp}(\chi-1)=\chi_{\perp}\left(\mathrm{T}_{\mathrm{e} 0}{ }^{\prime} / \mathrm{T}_{0 / 0}{ }^{\prime}-1\right)$ measures the enhanced radial heat diffusivity due to the parallel transport across the island. From Eq. (A11) is found that 


$$
\chi_{r}(r)=\chi_{\perp} \frac{w^{4}}{8 w_{c}^{4}} g(z)=\chi_{\|} \frac{b_{1 r}^{2}\left(r_{s}\right)}{2 B_{0 t}^{2}} g(z) .
$$

Eq. (A12) gives the enhanced radial transport due to a single island in the limit $\mathrm{w} / \mathrm{w}_{\mathrm{c}}<<1$, which agrees with the numerical result as shown in Fig. 7.

When there are magnetic perturbations of different helicities, and each individual island width $\mathrm{w}_{\mathrm{k}}$ calculated from the conventional single island formula is smaller than its corresponding critical island width $\mathrm{w}_{\mathrm{c}, \mathrm{k}}$, then in the lowest order $\mathrm{T}_{\mathrm{k}}$ is found to be determined by an equation like Eq. (A6), and one obtains $\mathrm{T}_{\mathrm{k}}=-2^{-5 / 2}\left(\mathrm{~W}_{\mathrm{k}}{ }^{2} / \mathrm{W}_{\mathrm{c}, \mathrm{k}}\right) \mathrm{T}_{\mathrm{s}}{ }_{\mathrm{f}} \mathrm{f}\left(\mathrm{z}_{\mathrm{k}}\right)$, where $\mathrm{z}_{\mathrm{k}}=2^{3 / 2}(\mathrm{r}--$ $\left.\mathrm{r}_{\mathrm{s}, \mathrm{k}}\right) / \mathrm{W}_{\mathrm{c}, \mathrm{k}}$ and $\mathrm{r}_{\mathrm{s}, \mathrm{k}}$ is the minor radius of the rational surface of the $k$ th component. A similar analysis to Eqs. (A7)-(A12) leads to the quasi-linear result

$$
\delta T(z)=-\sum \frac{w_{k}^{4}}{8 w_{c, k}^{4}} T_{0}^{\prime}(r) g_{k}\left(z_{k}\right)
$$

and

$$
\chi_{r}(r)=\sum \chi_{\perp} \frac{w_{k}^{4}}{8 w_{c, k}^{4}} g_{k}\left(z_{k}\right)=\sum \chi_{\|} \frac{b_{r, k}^{2}}{2 B_{0 t}^{2}} g_{k}\left(z_{k}\right),
$$

where the subscript $\mathrm{k}$ corresponds to the $k$ th component of magnetic perturbations, the summation is over $\mathrm{k}$ for including contribution from all components. Eq. (A14) agrees with the numerical results as shown in Figs. 16-19 for $\mathrm{w}_{\mathrm{k}}<\mathrm{w}_{\mathrm{c}, \mathrm{k}}$.

Eq. (A14) reduces to Eq. (12) in the limit $\mathrm{z}_{\mathrm{k}}=0$ except for a factor $2 \delta\left(\mathrm{m}_{\mathrm{k}} / \mathrm{q}-\mathrm{n}_{\mathrm{k}}\right)$. The function $\mathrm{g}_{\mathrm{k}}\left(\mathrm{z}_{\mathrm{k}}\right)$ in Eq. (A14), however, indicates the role of $\mathrm{W}_{\mathrm{c}, \mathrm{k}}$. Since $\mathrm{g}_{\mathrm{k}}\left(\mathrm{z}_{\mathrm{k}}\right)$ approaches zero as $\mathrm{z}_{\mathrm{k}}>2$, only these magnetic field perturbations with their rational surfaces being sufficiently close to the observation location $r$ have a significant contribution to $\chi_{r}$, and $\chi_{r}$ is approximately determined by the additive effects of these individual islands satisfying $\mathrm{z}_{\mathrm{k}}<2$. This is different from Eqs. (9)-(12) in which the $\mathrm{D}_{\mathrm{M}}$ includes the contribution from all resonant magnetic perturbations. 
The valid regime of Eq. (A14) is also very different from that of Eq. (12). To be self-consistent Eq. (A14) requires $\sum \Lambda_{\mathrm{k}} \mathrm{g}_{\mathrm{k}}\left(\mathrm{z}_{\mathrm{k}}\right)<2$, where $\Lambda_{\mathrm{k}}=\left(\chi_{\|} / \chi_{\perp}\right)\left(\mathrm{b}_{\mathrm{r}, \mathrm{k}} / \mathrm{B}_{0 \mathrm{t}}\right)^{2}$. While Eq. (12) is valid for $\tau_{\perp}<\tau_{\|}<\tau_{\mathrm{k}}$, leading to $\sum \Lambda_{\mathrm{k}}<\mathrm{A}_{1}$ and $\sum\left(\mathrm{b}_{\mathrm{r}, \mathrm{k}} / \mathrm{B}_{0 \mathrm{t}}\right)^{2}<\mathrm{A}_{2}$, where $\mathrm{A}_{1}=\left(\mathrm{k}_{\perp} \mathrm{L}_{0}\right)^{2} \sum\left(\mathrm{b}_{\mathrm{r}, \mathrm{k}} / \mathrm{B}_{0 \mathrm{t}}\right)^{2}$ and $\mathrm{A}_{2}=\left[\mathrm{RqL}_{\mathrm{q}} /\left(\mathrm{mL}_{0}^{2}\right)\right]^{2}$. For typical tokamak parameters $\mathrm{R} / \mathrm{a}=3, \mathrm{q}=2, \mathrm{~m}=5, \mathrm{~L}_{\mathrm{q}}=\mathrm{a}, \mathrm{r}_{\mathrm{s}}=0.8 \mathrm{a}$, and $\sum b_{r, k} / B_{0 t}=10^{-4}$, one finds $A_{1}=5.5 \times 10^{-6}$ and $A_{2}=1.8 \times 10^{-4}$. Eq. (A14) is valid for a much higher $\chi_{\|} / \chi_{\perp}$ than Eq. (12) since $A_{1} \sim 10^{-6}$ is very small. The condition $\sum\left(\mathrm{b}_{\mathrm{r}, \mathrm{k}} / \mathrm{B}_{0 \mathrm{t}}\right)^{2}<\mathrm{A}_{2}$ is usually satisfied for the magnetic perturbations in tokamak plasmas [2-8].

The Kadomsev-Pogutse regime, Eq. (11), is valid for $\tau_{\|}<\tau_{\perp}<\tau_{\mathrm{k}}$, corresponding to $\mathrm{A}_{1}<\sum \Lambda_{\mathrm{k}}<\mathrm{A}_{3}$, where $\mathrm{A}_{3}=\left[\left(\sum \mathrm{b}_{\mathrm{r}, \mathrm{k}} / \mathrm{B}_{0 \mathrm{t}}\right)\left(\mathrm{Rq}^{2} \mathrm{~L}_{\mathrm{q}}^{2} \mathrm{~m}\right) /\left(\pi \mathrm{r}^{3}\right)\right]^{2 / 3}$. For the above parameters one finds $\mathrm{A}_{3}=0.013$. Therefore, the valid regime of Eq. (A14) covers the Kadomsev-Pogutse regime, indicating that Eqs. (11) and (12) are problematic. The Rechester-Rosenbluth regime, Eq. (10), is valid for $\tau_{k}<\tau_{\perp}$ (in addition to $\tau_{\|}<\tau_{k}$ ), leading to $\sum \Lambda_{k}>A_{3}$. It is seen that the valid regime of Eq. (A14) could well extend into the Rechester-Rosenbluth regime, especially for the case with smaller perturbation amplitudes in a large magnetic shear region. 


\section{Reference}

[1] H. P. Furth, J. Killen, and M. N. Rosenbluth, Phys. Fluids 6, 459(1963).

[2] J. Wesson, Tokamaks (Clarendon Press, Oxford, 1987).

[3] J. A. Wesson, R.D. Gill, M. Hugon, F.C. Schüller, et al., Nucl. Fusion 29, 641(1989).

[4] Z. Chang, J. D. Callen, E. D. Fredrickson, et al., Phys. Rev. Letts, 74, 4663(1995).

[5] H. Zohm, G. Gantenbein, A. Gude, S. Günter, et al., Nucl. Fusion 41, 197(2001).

[6] S. Günter,A. Gude, M. Maraschek, S. Sesnic, H. Zohm, et al., Phys. Rev. Letters, 87, 275001(2001).

[7] S. Günter, A. Gude, M. Maraschek, Q. Yu and the ASDEX Upgrade Team, Plasma Phys. Control. Fusion, 41, 767(1999).

[8] R. J. La Haye, L. L. Lao, E. J. Strait, and T. S. Taylor, Nucl. Fusion 37, 397(1997).

[9] Q. Yu, S. Günter and K. Lackner, Phys. Plasmas 11, 140(2004).

[10] N. T. Gladd, J. F. Drake, C. L. Zhang, and C. S. Liu, Phys. Fluids 23, 1182(1980).

[11] Q. Yu, S. Günter and Bruce D. Scott, Phys. Plasmas 10, 797(2003).

[12] Q. Yu, S. Günter, K. Lackner, et al., Nucl. Fusion, 40, 2031 (2000).

[13] E. Strumberger, J. Nucl. Materials, 266-269, 1207(1999).

[14] Ph. Ghendrih, A. Grosman and H. Capes, Plasma Phys. Control. Fusion, 38, 1653(1996).

[15] Z. Chang and J.D. Callen, Nucl. Fusion 30, 219(1990).

[16] Richard Fitzpatrick, Phys. Plasmas 2, 825(1995).

[17] A. B. Rechester and M. N. Rosenbluth, Phys. Rev. Letters, 40, 38(1978).

[18] T. H. Stix, Nucl. Fusion 18, 353(1978).

[19] B. B. Kadomtsev and O. P. Pogutse, in Plasma Physics and Controlled Fusion Research 1978, Proceedings of 7th International Conference, Innsbruck (international Atomic Energy Agency, Vienna, 1979), Vol. 1, P. 649.

[20] J. A. Krommers, C. Oberman and R. G. Kleva, J. Plasma Physics, 30, 11(1983). 
[21] A. B. Rechester, M. N. Rosenbluth and R.B. White, Phys. Rev. Letters, 42, 1247(1979).

[22] A. H. Boozer and R.B. White, Phys. Rev. Letters, 49, 786(1979).

[23] E. Vanden Eijnden and R. Balescu, Phys. Plasmas 4, 270(1997).

[24] M. de Rover, A. M. R. Schilham, A. Montvai, and N. J. Lopes Cardozo, Phys. Plasmas 6, 2443(1999).

[25] R. J. Bickerton, Plasma Phys. Control. Fusion, 39, 339(1997).

[26] P. C. Liewer, Nucl. Fusion 25, 543(1985).

[27] S. Günter, Q. Yu, J. Krüger and K. Lackner, J. Comp. Phys. 209, 35(2005).

[28] Z. Chang and J.D. Callen, Phys. Fluids B4, 1167(1992).

[29] G. W. Hammett and F. W. Perkins, et al., Phys. Rev. Letters, 64, 3019(1990).

[30] Q. Yu, S. Günter, G. Giruzzi, K. Lackner, and M. Zabiego, Phys. Plasmas 7, 312(2000).

[31] R.C. Wolf, W. Biel, M.F.M. de Bock, et al., Nucl. Fusion 45, 1700(2005),

[32] T. E. Evans, R. A. Moyer, P.R. Thomas, et al., Phys. Rev. Letters, 92, 235003(2004).

[33] A. Gude, S. Günter, S. Sesnic, ASDEX Upgrade Team, Nucl. Fusion 39, 127(1999).

[34] E. D. Fredrickson, Phys. Plasmas 9, 548(2002). 


\section{Caption}

Fig. 1 Radial profiles of $\mathrm{T}_{\mathrm{e} 0}$ (dotted curve), $\mathrm{T}_{0 / 0}$ and the $\mathrm{T}_{\mathrm{e}}$ along the line passing through the island's o-point and $\mathrm{x}$-point with $\mathrm{w}_{3 / 2}=0.0452 \mathrm{a}, \mathrm{r}_{3 / 2}=0.604 \mathrm{a}$ and $\chi_{\|} / \chi_{\perp}=10^{10} . \mathrm{T}_{0 / 0}{ }^{\prime}$ is same as $\mathrm{T}_{\mathrm{e} 0}{ }^{\prime}$ for $\mathrm{r}<\mathrm{r}_{-}$, and $\mathrm{T}_{0 / 0}$ is the same as $\mathrm{T}_{\mathrm{e} 0}$ for $\mathrm{r}>\mathrm{r}_{+}$.

Fig. 2 Corresponding to Fig. 1, radial profiles of the $m / n=3 / 2,6 / 4,9 / 6$, and 12/8 components of $\mathrm{T}_{\mathrm{e}}$. High harmonic temperature perturbations are localized in the island region, and their amplitudes decrease for a larger $m(n)$.

Fig. 3 Radial profiles of $\mathrm{T}_{\mathrm{e} 0}$ (solid) and the $\mathrm{T}_{0 / 0}$ for $\chi_{\|} / \chi_{\perp}=10^{12}, 10^{10}, 10^{8}$ and $10^{6}$. For a low $\chi_{\|} / \chi_{\perp}, \mathrm{T}_{0 / 0}$ is close to $\mathrm{T}_{\mathrm{e} 0}$. A larger $\chi_{\|} / \chi_{\perp}$ leads to a more flattening local $\mathrm{T}_{0 / 0}$ profile.

Fig. 4 Corresponding to Fig. 3, radial profiles of $\mathrm{T}_{3 / 2}$ for $\chi_{\|} / \chi_{\perp}=10^{10}, 10^{8}$ and $10^{6}$. For $\chi_{\|} / \chi_{\perp}=10^{6}, \mathrm{~T}_{3 / 2}$ and $\mathrm{T}_{3 / 2}{ }^{\prime}$ are small in the island region. A larger $\chi_{\|} / \chi_{\perp}\left(=10^{8}\right)$ leads to a larger $\mathrm{T}_{3 / 2}$ and $\mathrm{T}_{3 / 2}^{\prime}$. For $\chi_{\|} / \chi_{\perp}=10^{10}, \mathrm{~T}_{3 / 2}$ profile becomes flattening near the rational surface.

Fig. 5 Corresponding to Fig. 3, radial profiles of $\chi$ for $\chi_{\|} / \chi_{\perp}=10^{10}, 10^{8}$ and $10^{6}$. $\chi$ has a maximal value at $r_{3 / 2}$ and a width about the island width. A larger $\chi_{\|} / \chi_{\perp}$ leads to a larger $\chi$.

Fig. $6 \log (\chi-1)_{\max }$ versus $\log \left(\chi_{\|} / \chi_{\perp}\right)$ for the $3 / 2$ island (solid) and the $4 / 3$ island (dotted). $\mathrm{w}_{3 / 2}=\mathrm{w}_{\mathrm{c}, 3 / 2}$ leads to $\log \left(\chi_{\|} / \chi_{\perp}\right)_{\mathrm{c}}=6.6$. $(\chi-1)_{\max } \propto \chi_{\|} / \chi_{\perp}$ for $\chi_{\|} / \chi_{\perp}<\left(\chi_{\|} / \chi_{\perp}\right)_{\mathrm{c}}$ but $(\chi-1)_{\max } \propto\left(\chi_{\|} / \chi_{\perp}\right)^{1 / 2}$ in the opposite limit. There is a transition region around $10\left(\chi_{\|} / \chi_{\perp}\right)_{c}$ or $w_{3 / 2}=1.8 \mathrm{w}_{\mathrm{c}, 3 / 2}$.

Fig. 7 Numerical results of $\log (\chi-1)_{\max }$ versus $\log \left(\mathrm{w} / \mathrm{w}_{\mathrm{c}}\right)^{2}$ for a $3 / 2$ island with $\chi_{\|} / \chi_{\perp}=10^{10}$ (solid). Here in $\left(\mathrm{w} / \mathrm{w}_{\mathrm{c}}\right)^{2}$ only the island width $\mathrm{w}$ veries. $(\chi-1)_{\max } \propto \mathrm{w}^{4}$ for $\mathrm{w} / \mathrm{w}_{\mathrm{c}}<<1$ but $(\chi-1)_{\max } \propto \mathrm{w}^{2}$ for $\mathrm{w} / \mathrm{w}_{\mathrm{c}}>>1$. The transition region between these two limits is around $\mathrm{w} / \mathrm{w}_{\mathrm{c}}=1.8$. The doted curve shows the analytical results of Eq. (A12).

Fig. $8 \Delta \beta / \beta_{0}$ versus $\log \left(\chi_{\|} \chi_{\perp}\right)$ for the $3 / 2$ island with $w_{3 / 2}=0.0452 \mathrm{a}$ (solid). $\Delta \beta$ increases with $\log \left(\chi_{\|} / \chi_{\perp}\right)$ from $\left(\chi_{\|} / \chi_{\perp}\right)_{\mathrm{c}}=3.8 \times 10^{6}$ up to $10^{10}\left(\mathrm{w}_{3 / 2} / \mathrm{w}_{\mathrm{c}}=7.2\right)$. For $\chi_{\|} / \chi_{\perp}>10^{10}, \Delta \beta$ saturates. 
The $\Delta \beta / \beta_{0}$ due to a $4 / 3$ island (dotted) is larger for low $\chi_{\|} / \chi_{\perp}$ due to its smaller $\mathrm{w}_{\mathrm{c}}$. For high $\chi_{\|} / \chi_{\perp}$, the $\Delta \beta / \beta_{0}$ due to the $3 / 2$ island is larger because $r_{3 / 2}>r_{4 / 3}$ and $w_{3 / 2}>>w_{c}$.

Fig. 9 The magnetic surface on the $r-\theta$ plan at $\phi=0$ for $\psi_{0}=10^{-4}$. An secondary $m / n=7 / 5$ island exists in addition to the $3 / 2$ and $4 / 3$ islands.

Fig. 10 Corresponding to Fig. 9, the temperature contour for $\chi_{\|} / \chi_{\perp}=10^{12}$. There is an $\mathrm{m} / \mathrm{n}=7 / 5$ island in the temperature contour in agreement with Fig. 9.

Fig. 11 Same as Fig. 9 but for $\psi_{0}=9 \times 10^{-4}$. The local field becomes stochastic.

Fig. 12 Corresponding to Fig. 11, radial profiles of $\mathrm{T}_{\mathrm{e} 0}$ (dot-dashed) and the $\mathrm{T}_{0 / 0}$ for $\chi_{\|} / \chi_{\perp}=10^{10}, 10^{8}$ and $10^{6}$. A larger $\chi_{\|} / \chi_{\perp}$ leads to a more flattening local $\mathrm{T}_{0 / 0}$ profile.

Fig. 13 Corresponding to Fig. 12, radial profiles of $\log (\chi-1)$. For $\chi_{\|} / \chi_{\perp}=10^{8}, \chi$ only peaks around the $\mathrm{q}=3 / 2$ and $4 / 3$ surfaces. For $\chi_{\|} / \chi_{\perp}=10^{10}$, additional peaks appear due to the ergodicity, and $\chi$ is about 5 times larger than that for a single island.

Fig. 14 Radial profiles of $\mathrm{T}_{\mathrm{e} 0}$ and the $\mathrm{T}_{0 / 0}$ for $\psi_{0}=2 \times 10^{-4}, 5 \times 10^{-4}$ and $9 \times 10^{-4}$ with $\chi_{\|} / \chi_{\perp}=10^{10}$. With the increase of the island width, the local $\mathrm{T}_{0 / 0}^{\prime}$ decreases.

Fig. $15 \Delta \beta / \beta_{0}$ (solid) and $\Delta \beta_{1} / \beta_{0}=\left(\Delta \beta_{3 / 2}+\Delta \beta_{4 / 3}\right) \beta_{0}$ (dotted) versus $\log \left(\chi_{\|} / \chi_{\perp}\right)$ with $\psi_{0}=9 \times 10^{-4}$. For low $\chi_{\|} / \chi_{\perp}, \Delta \beta=\Delta \beta_{1}$ as predicted by Eq. (A13). For high $\chi_{\|} / \chi_{\perp}, \Delta \beta>\Delta \beta_{1}$, showing the enhanced transport due to ergodicity.

Fig. $16 \log (\chi-1)$ at $r=0.587$ a versus $\log \left(\chi_{\|} / \chi_{\perp}\right)$ for $\psi_{0}=9 \times 10^{-4}$ (solid). For low or high $\chi_{\|} / \chi_{\perp},(\chi-1) \propto \chi_{\|} / \chi_{\perp}$. There is a transition region around $\chi_{\|} / \chi_{\perp}=3 \times 10^{7}\left(\mathrm{w}_{3 / 2}=1.7 \mathrm{w}_{\mathrm{c}, 3 / 2}\right)$. The dotted curve shows $\log \left(\chi_{3 / 2}+\chi_{4 / 3}-2\right)$, where $\chi_{3 / 2}\left(\chi_{4 / 3}\right)$ is the $\chi$ for a single $3 / 2$ (4/3) island. The two curves are the same for $\chi_{\|} / \chi_{\perp}<3 \times 10^{7}$ as predicted by Eq. (A14). 
Fig. $17 \kappa$ versus $\log \left(\chi_{\|} \chi_{\perp}\right)$ for $\psi_{0}=1 \times 10^{-4}, 6 \times 10^{-4}, 9 \times 10^{-4}, 2.1 \times 10^{-3}$, and $3.0 \times 10^{-3} . \kappa$ is the same with different $\psi_{0}$ for low $\chi_{\|} / \chi_{\perp}$ as predicted by Eq. (A14). For high $\chi_{\|} / \chi_{\perp}, \kappa$ oscillates for $\psi_{0}<2 \times 10^{-3}$ but approaches a nearly steady value for larger $\psi_{0}$.

Fig. $18 \kappa$ versus $\log \left(\chi_{\|} / \chi_{\perp}\right)$ for $\psi_{0}=3.0 \times 10^{-3}, 4.5 \times 10^{-3}$ and $6.0 \times 10^{-3}$, with $\mathrm{L}_{\mathrm{q}}=0.1 \mathrm{a}$, $r_{3 / 2}=0.595 \mathrm{a}$ and $r_{4 / 3}=0.583 \mathrm{a}$. Other parameters are the same as those for Fig. 17. The dotted curve is the result with $\mathrm{L}_{\mathrm{q}}=0.3 \mathrm{a}$ and $\psi_{0}=3.0 \times 10^{-3} . \kappa$ becomes smaller for a larger magnetic shear. The dashed curve is obtained from Eq. (9) for $\psi_{0}=6.0 \times 10^{-3}$.

Fig. $19 \kappa$ versus $\log \left(\chi_{\|} / \chi_{\perp}\right)$ with $\psi_{0}=9.0 \times 10^{-4}, 1.2 \times 10^{-3}, 1.8 \times 10^{-3}$, and $2.1 \times 10^{-3}$ and $\mathrm{m} / \mathrm{n}=30 / 19,30 / 20,30 / 21$, and $30 / 22$. The dotted curve is the result for only the $3 / 2$ and $4 / 3$ island and $\psi_{0}=2.1 \times 10^{-3} . \kappa$ is smaller for the 4 island case due to the smaller $\mathrm{w}_{\mathrm{c}}$.

Fig. $20 \quad f(z)$ and $g(z)$ versus $z$. $g(z)$ approaches zero for $z>2$. 


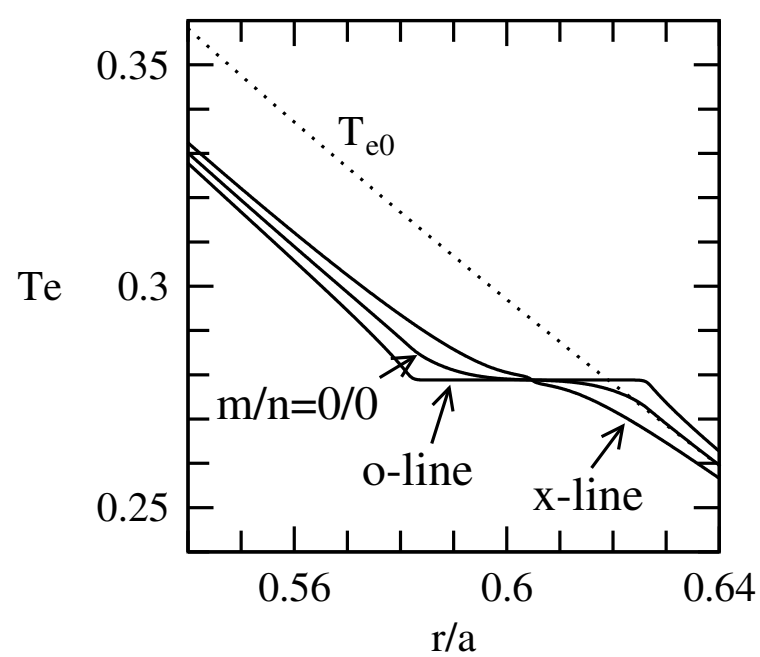

Fig. 1 


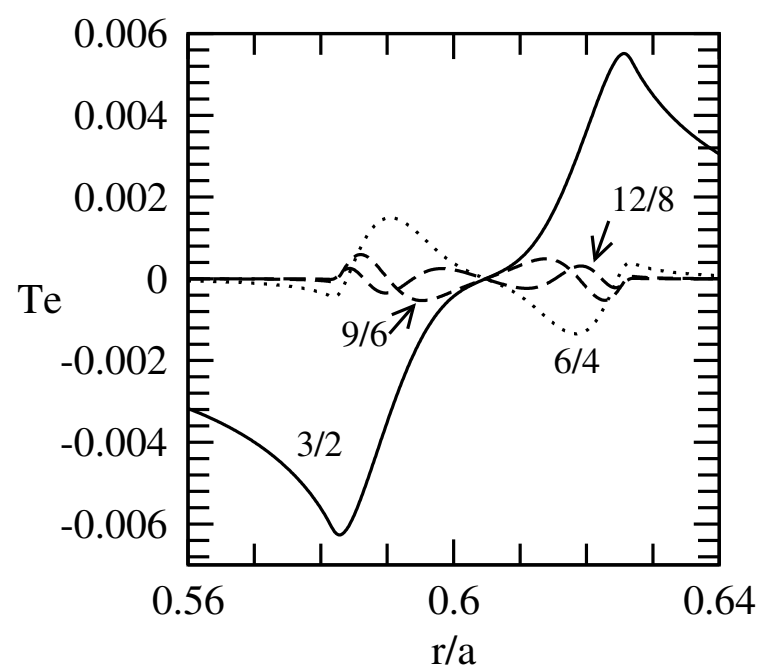

Fig.2 


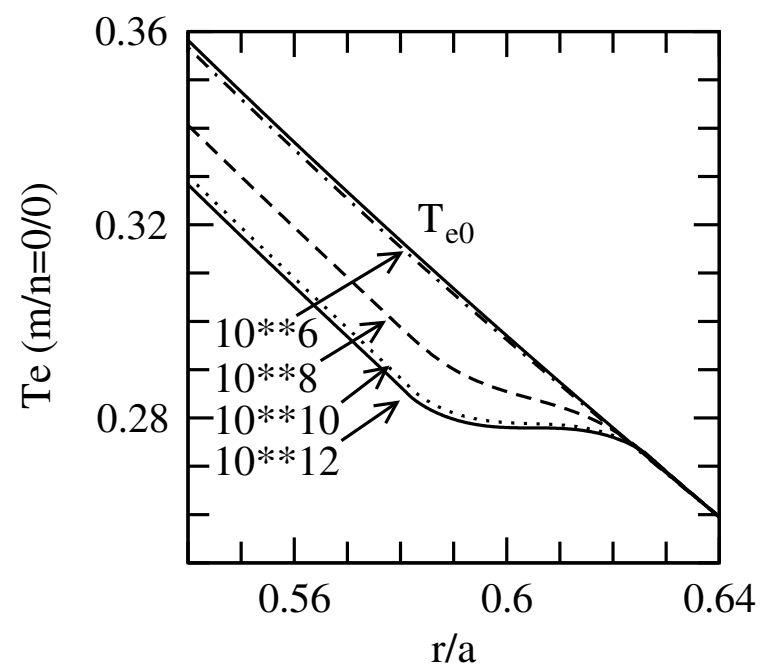

Fig. 3 


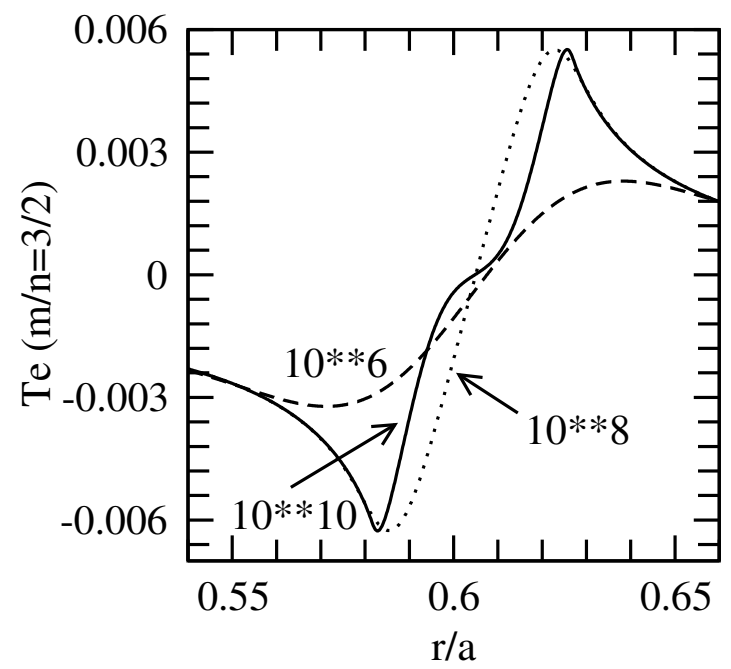

Fig. 4 


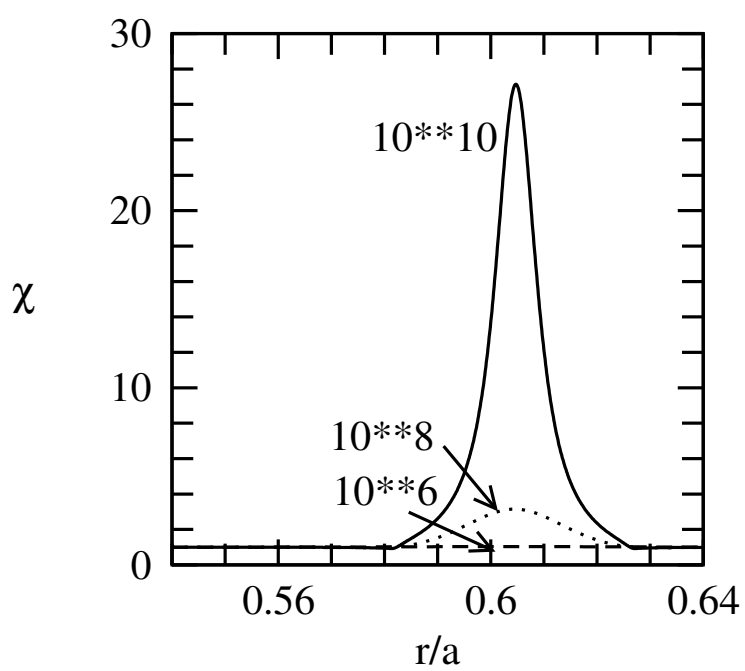

Fig. 5 


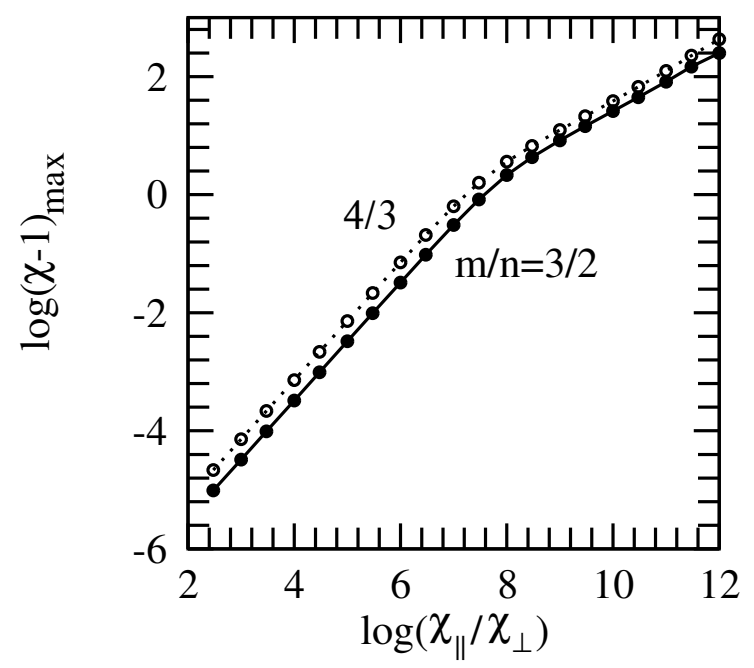

Fig. 6 


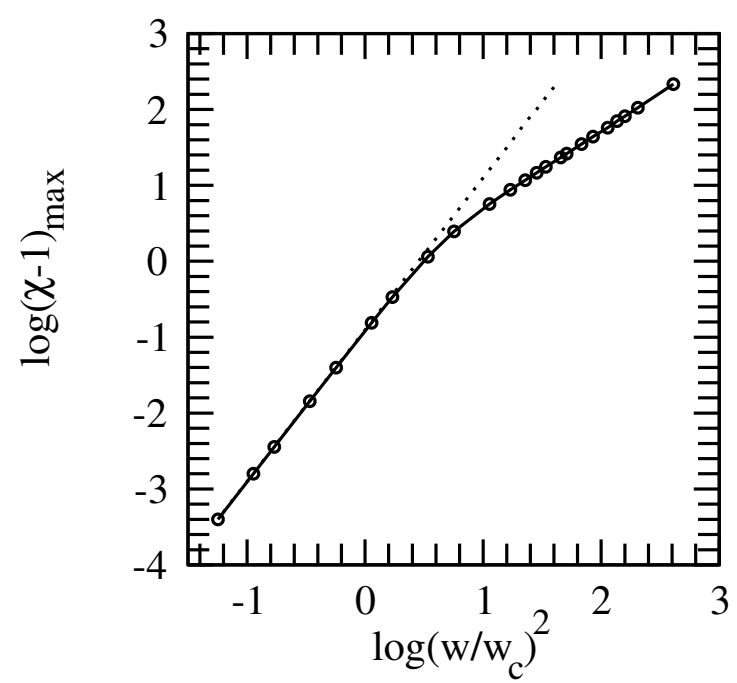

Fig. 7 


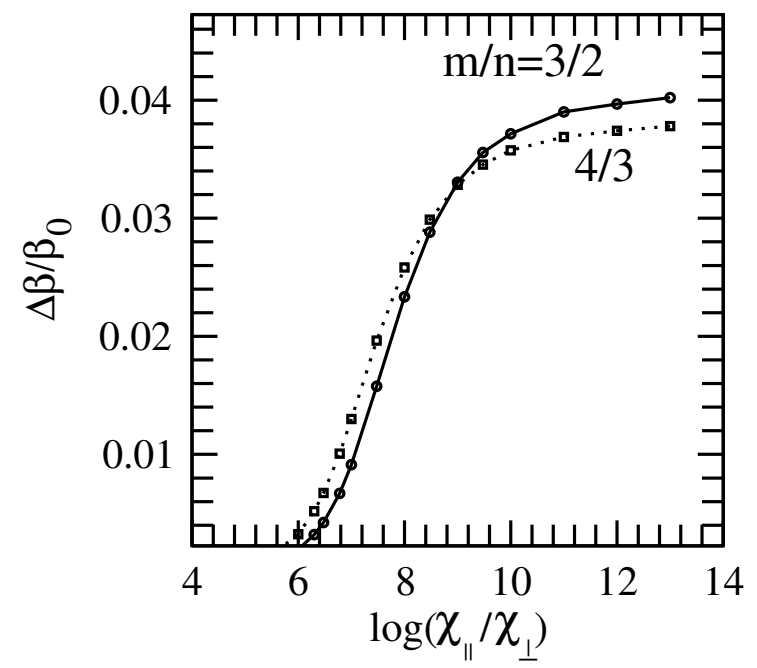

Fig. 8 


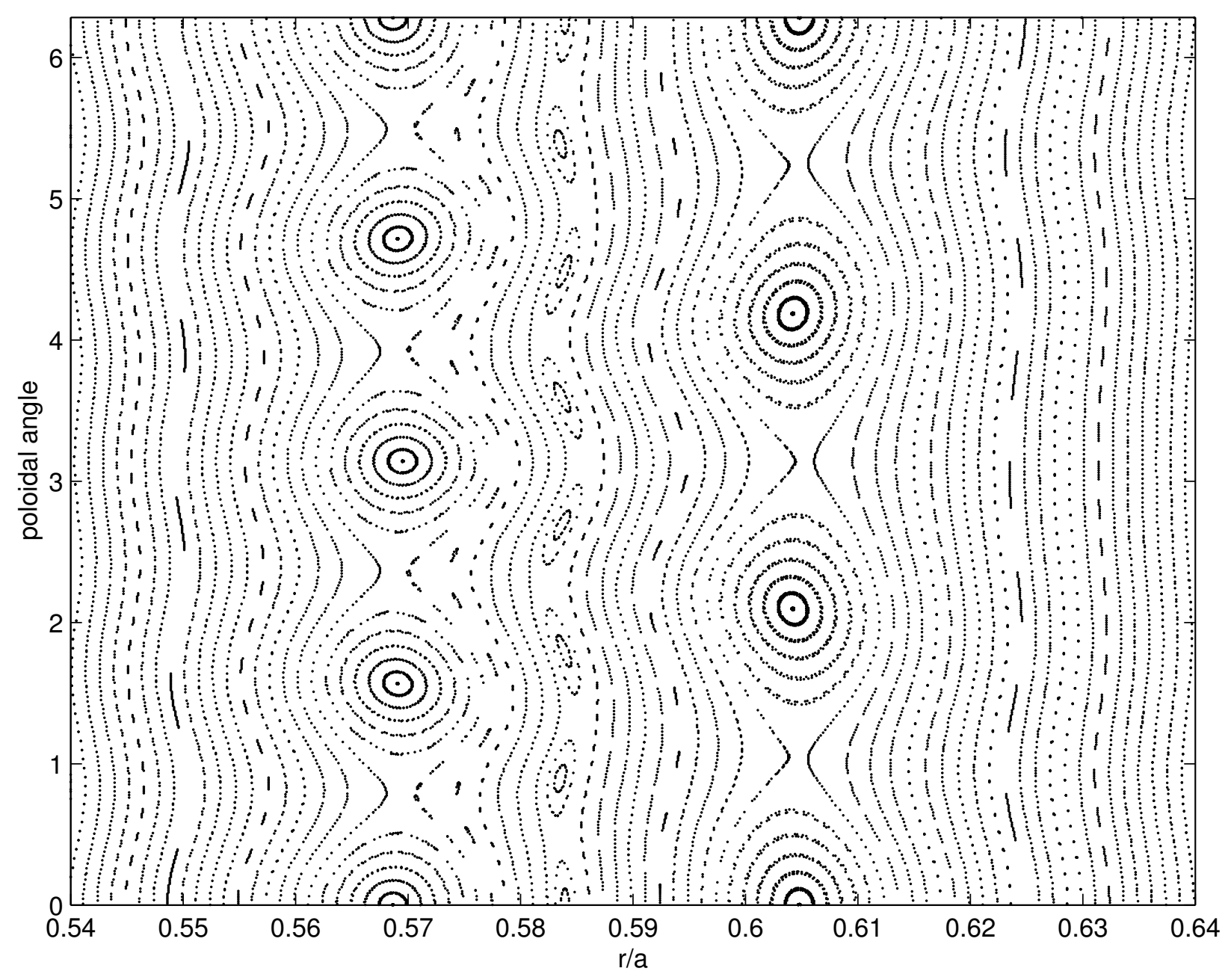

Fig. 9 


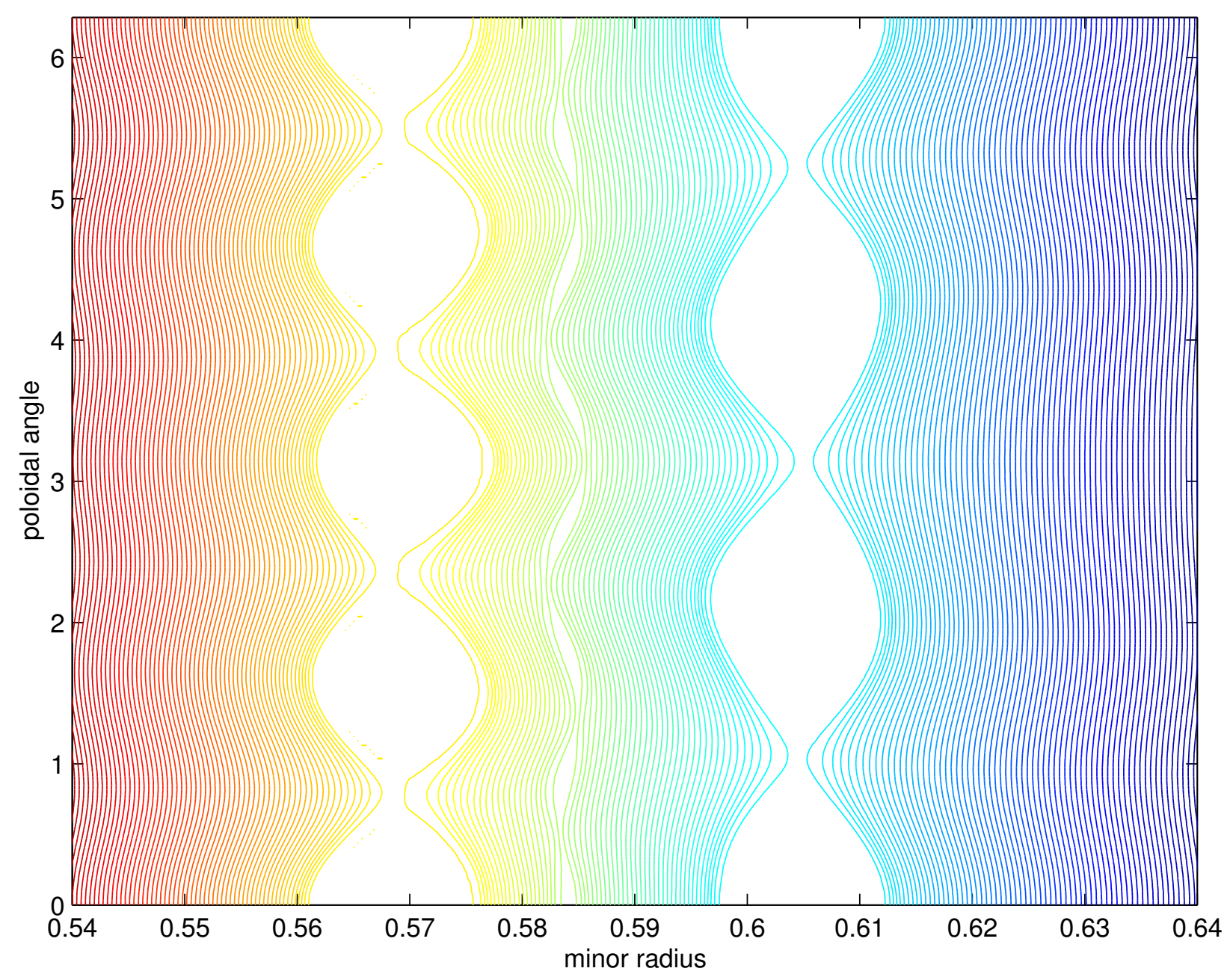

Fig. 10 


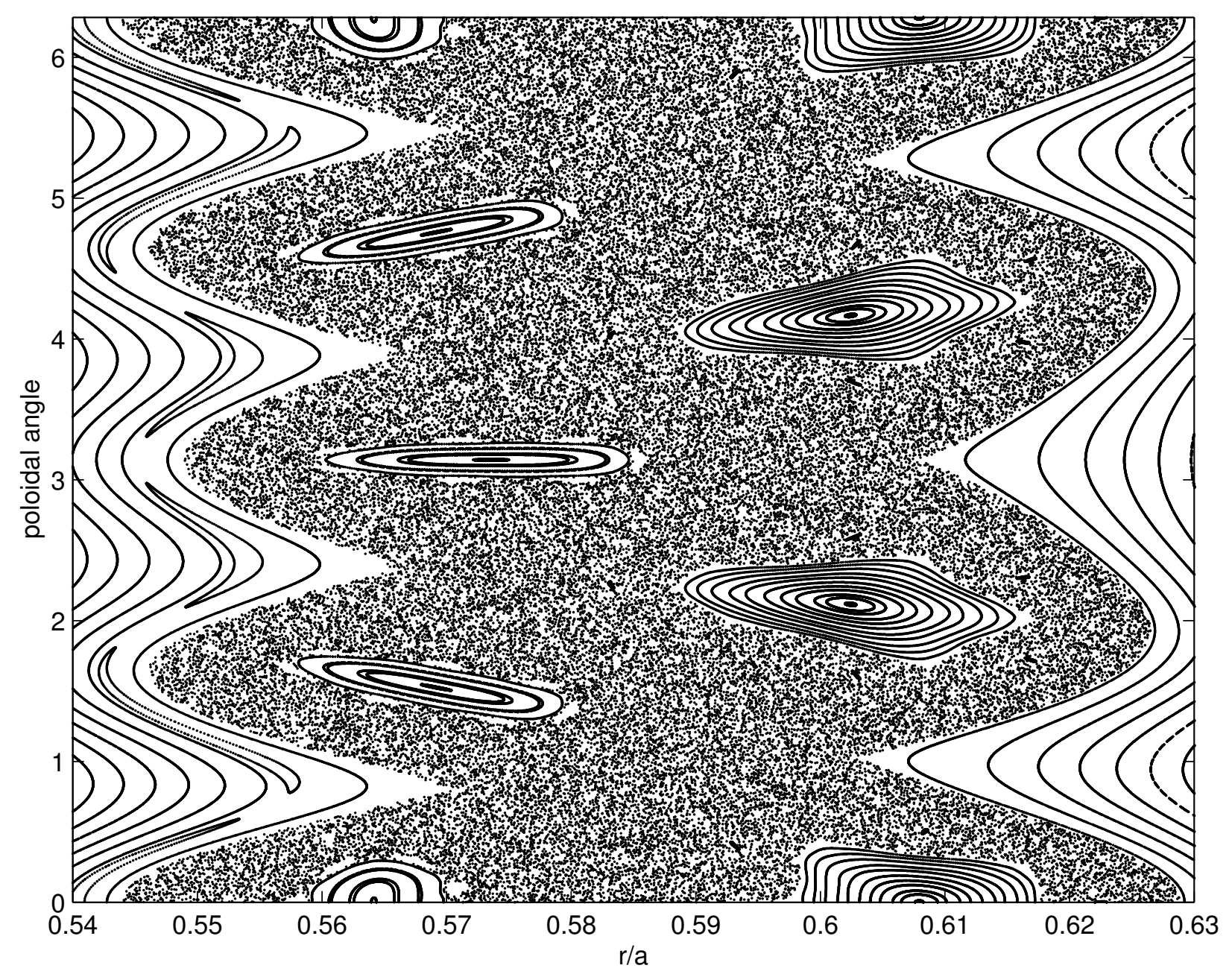

Fig. 11 


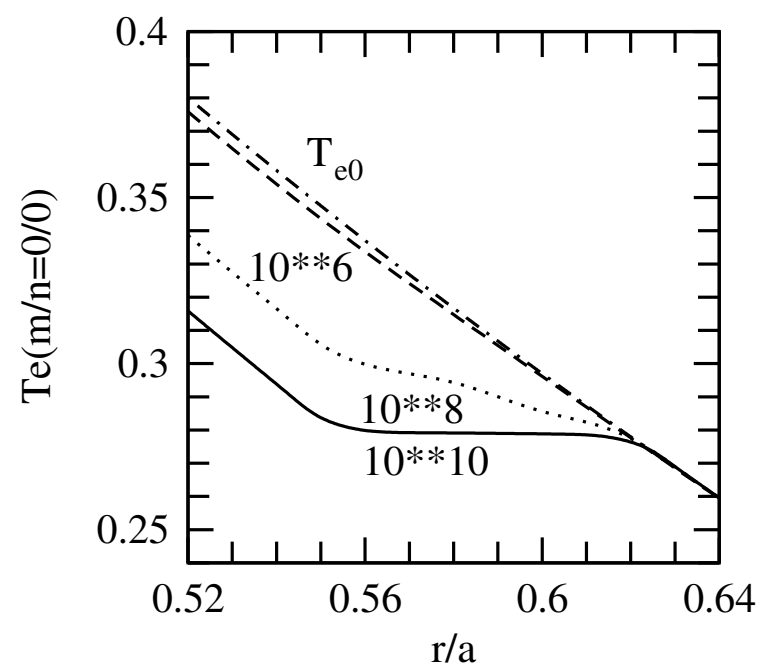

Fig. 12 


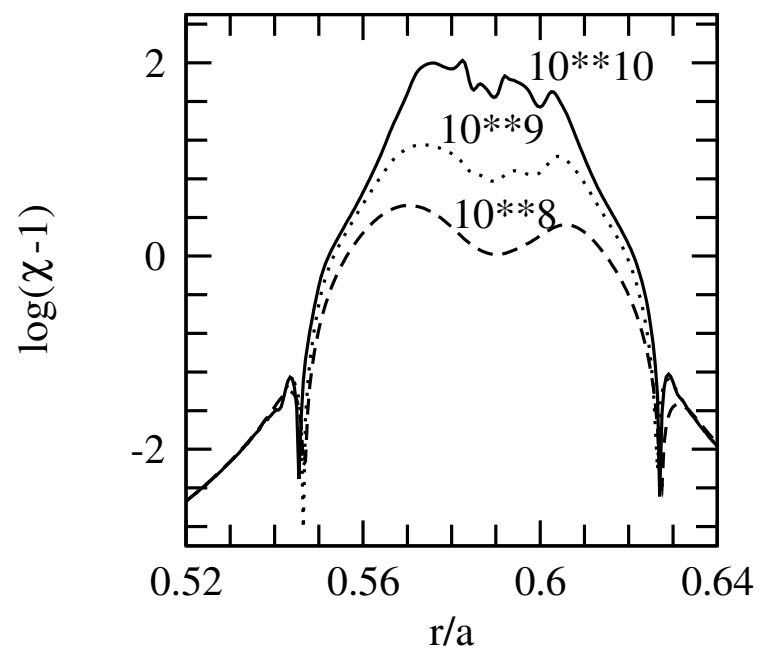

Fig. 13 


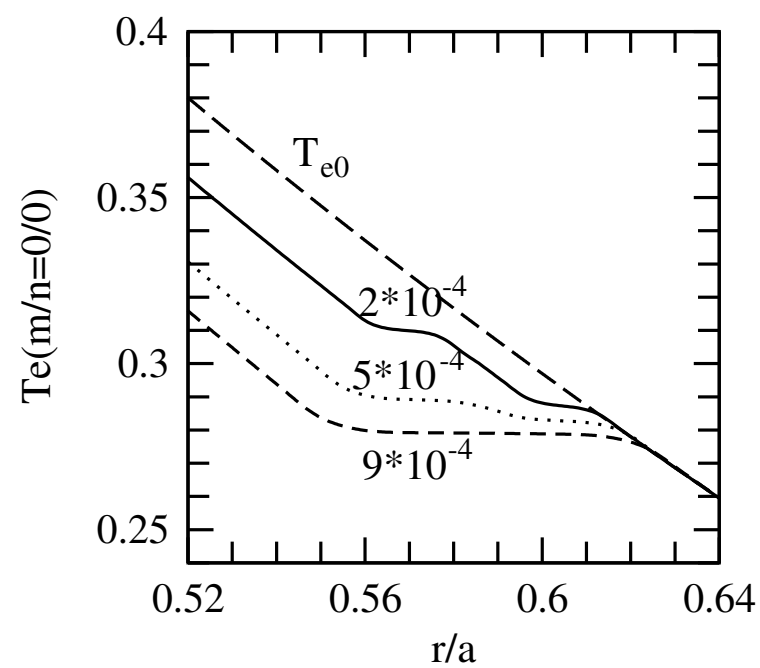

Fig. 14 


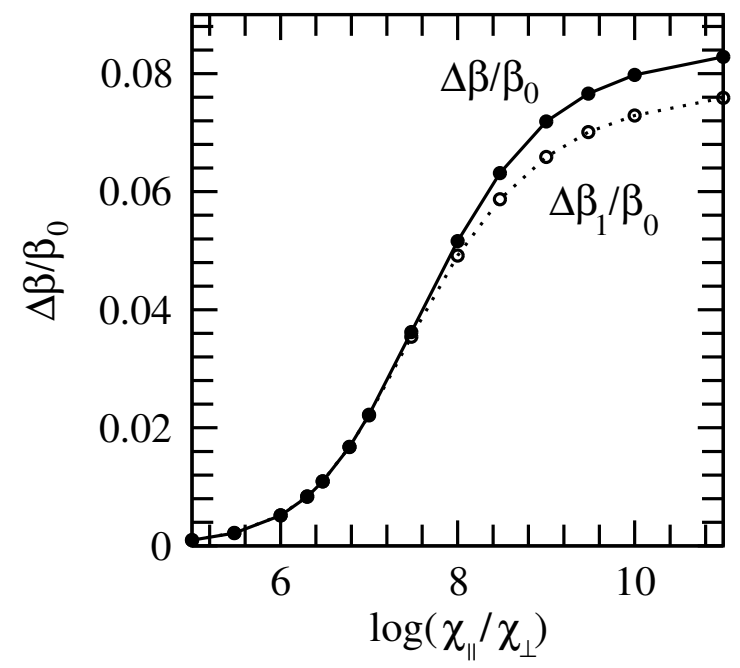

Fig. 15 


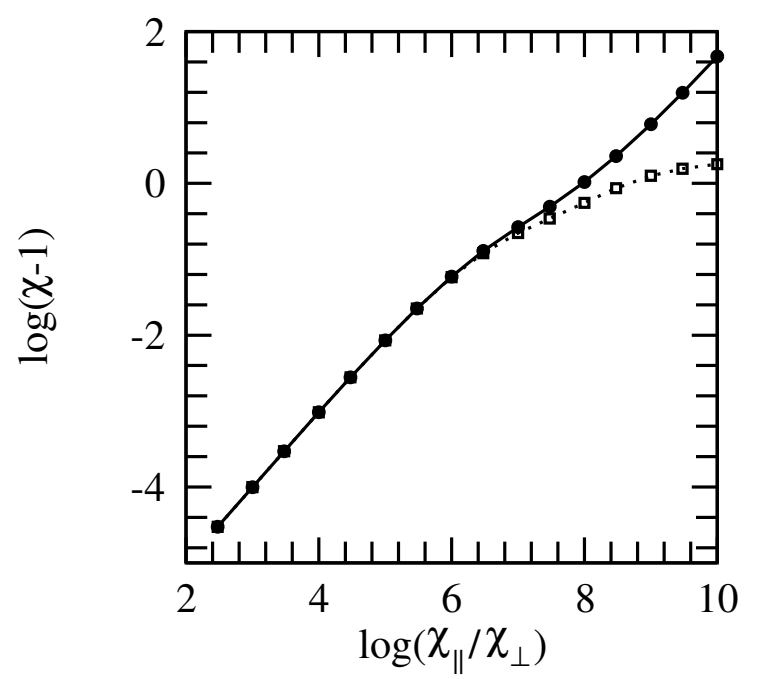

Fig. 16 


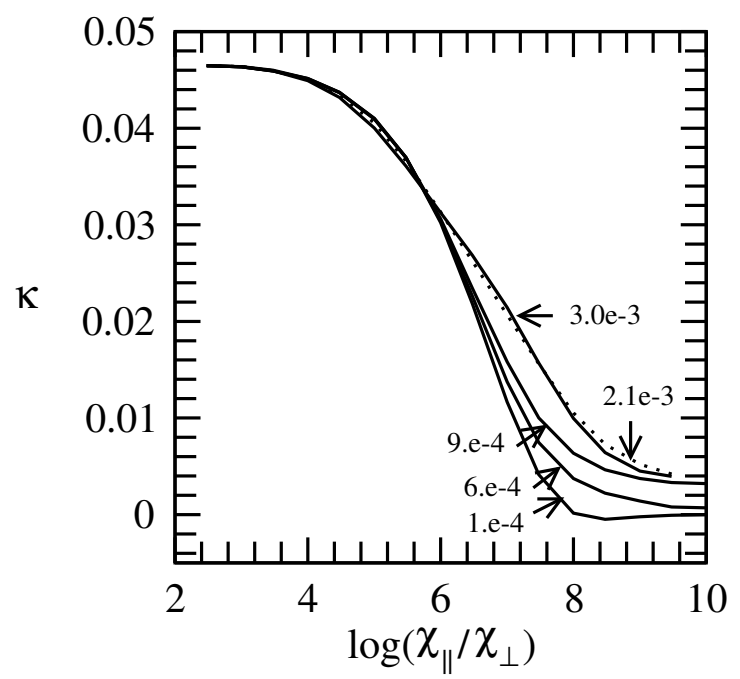

Fig. 17 


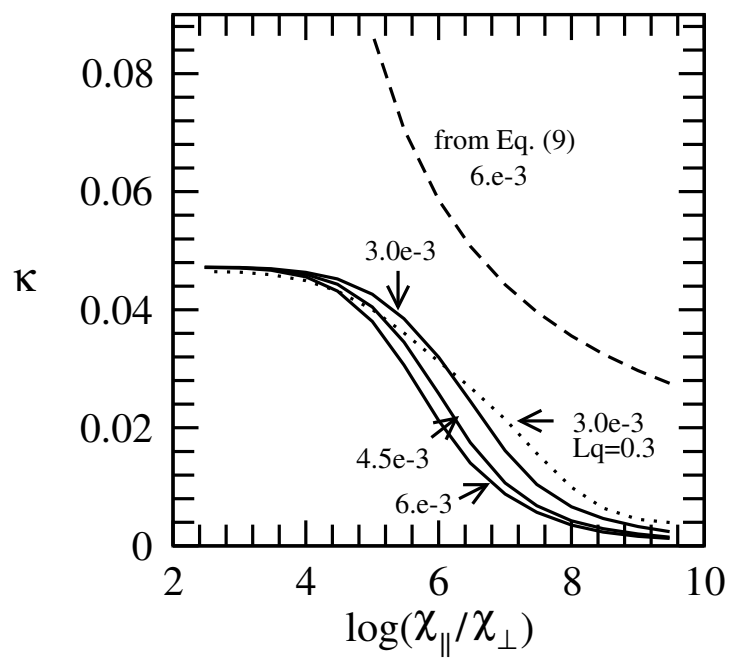

Fig. 18 


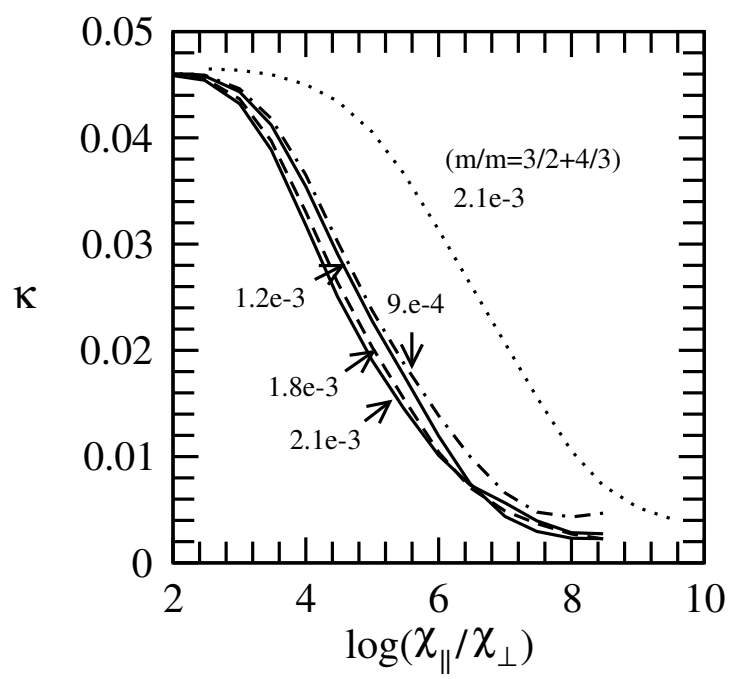

Fig. 19 


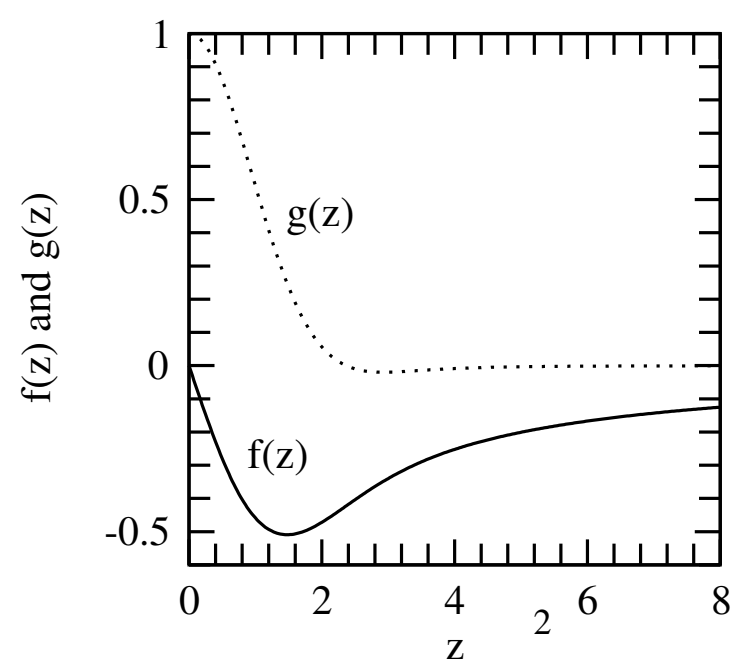

Fig. 20 